\title{
Maximum probability domains from Quantum Monte Carlo Calculations
}

\author{
Anthony Scemama \\ CERMICS, Ecole Nationale des Ponts et Chaussées, 6 et 8 avenue Blaise Pascal, \\ Cité Descartes - Champs sur Marne 77455 Marne la Vallée Cedex 2, France. \\ Michel Caffarel \\ Laboratoire de Chimie et Physique Quantiques, \\ CNRS-UMR 5626, IRSAMC Université Paul Sabatier, \\ 118 route de Narbonne 31062 Toulouse Cedex, France. \\ Andreas Savin \\ Laboratoire de Chimie Théorique, CNRS-UMR 7616, \\ Université Paris Pierre et Marie Curie, Case 137, \\ 4 place Jussieu 75252 Paris Cedex 05, France.
}

(Dated: July 28, 2006)

Dedicated to Professor Hans Georg von Schnering for his $75^{\text {th }}$ birthday. 


\begin{abstract}
Although it would be tempting to associate the Lewis structures to the maxima of the squared wave function $|\Psi|^{2}$, we prefer in this paper the use of domains of the threedimensional space which maximize the probability of containing opposite-spin electron pairs. We find for simple systems $\left(\mathrm{CH}_{4}, \mathrm{H}_{2} \mathrm{O}, \mathrm{Ne}, \mathrm{N}_{2}, \mathrm{C}_{2} \mathrm{H}_{2}\right)$ domains comparable to those obtained with the Electron Localization Function (ELF) or by localizing molecular orbitals. The different domains we define can overlap, and this gives an interesting physical picture of the floppiness of $\mathrm{CH}_{5}^{+}$and of the symmetric hydrogen bond in $\mathrm{FHF}^{-}$. The presence of multiple solutions has an analogy with resonant structures, as shown in the trans-bent structure of $\mathrm{Si}_{2} \mathrm{H}_{2}$. Correlated wave functions were used (MCSCF or Slater-Jastrow) in the Variational Quantum Monte Carlo framework.
\end{abstract}




\section{INTRODUCTION}

Lewis' concept of electron sharing producing electron pairs was extremely fruitful in chemistry, but its translation to quantum mechanics is not self-evident. Lewis himself was pessimistic.[1] In 1916, quantum mechanics was still in an embryonic stage, and Lewis found it "hardly likely that much progress can be made" using it.

From the present perspective, the obstacle is the connection between the high dimensionality of quantum mechanics (of the squared wave function, $|\Psi|^{2}$ ) and the three-dimensional space of the molecular structure. One approach is to reduce the dimensionality by averaging over the positions of all electrons except one (Atoms In Molecules[3]). One can also use other three-dimensional functions like localized orbitals[4], the Electron Localization Function[5] (ELF) or decompositions like in population analysis[6].

In Variational Quantum Monte Carlo (VMC), one works directly with $|\Psi|^{2}$. Thus, some of the techniques just mentioned are applicable, but inadequate. In this paper, we will try to show that there is a tool yielding an image close to that of Lewis and which is generally applicable to quantum mechanical methods including VMC.

In fact, Lewis was much concerned about the spatial arrangement of valence electrons. In his paper[1], he starts with a "cubical atom", the valence electrons being able to choose positions at the corners of a cube. This allows a maximum of eight electrons around an atom. As Lewis goes on with his argumentation, he tends to give it up due to "triple bonds for which the cubical structure offers no simple representation". Another problematic case he discusses is that of the Lithium atom where the octet rule (term later introduced by Langmuir[2]) is not satisfied. He recognizes that in such a case "the pair of electrons forms the stable group". Finally he suggests that for the $\mathrm{C}-\mathrm{C}$ bond the pairs of electrons around a carbon atom might be arranged tetrahedrally. Later, when the concept of electron spin was introduced, Linnett[10] considers that for each spin separately electrons arrange themselves tetrahedrally around the nucleus, and that the two tetrahedra are kept apart.

From our present perspective, $|\Psi|^{2}$ controls the spatial distribution of electrons. This has been exploited to return to the three-dimensional structure of molecules. It was suggested[7,

8] to simply select the most probable arrangement of valence electrons, i.e. the positions of the electrons which maximize $|\Psi|^{2}$. For example, in the $s p^{3}$ carbon atom, Artmann[7] shows that $|\Psi|^{2}$ is maximal when the electrons are localized at the vertices of a tetrahedron. In 
fact, it is quite easy to show that for perfectly localized orbitals, $\phi_{i}$, the maximum of the Slater determinant built from them corresponds to the arrangement where the electron pairs occupy the positions where $\left|\phi_{i}\right|$ take their largest value. $\left(\phi_{i}\right.$ are perfectly localized orbitals when $\int\left|\phi_{i}(\mathbf{r})\right|^{2}\left|\phi_{j}(\mathbf{r})\right|^{2} d \mathbf{r}=0, i \neq j$, or if $\phi_{i}(\mathbf{r}) \neq 0, \phi_{j}(\mathbf{r})=0$ for all $\left.j \neq i\right)$. Note that quantum mechanics allows two electrons to occupy the same infinitesimal volume element as long as they have opposite spins. In figure 1 the positions of the electron pairs at a maximum of the single determinant (Hartree-Fock) wave function are shown for the water molecule.

When the single-determinant approximation is overcome (correlation is taken into account), we find that electrons of a pair tend to separate: the maximum of the singledeterminant wave function is not a maximum of the correlated wave function. The maximum is achieved when the electrons of a pair occupy different positions in space. We see in figure 2 that in the water molecule the electrons of the $\mathrm{O}-\mathrm{H}$ bonds separate along the bonds, "pre-dissociate", while the electrons of the lone pairs separate in a direction perpendicular to the tetrahedral axis, similar to figure 5 in Lewis' paper[1]. Fulde [9] also noticed such effects in the correlated pair distributions. There are different equivalent maxima obtained by permutation of spins.

We now come to another concept of Lewis, namely that of tautomerism. Lewis assumes that in a given substance, "it must not be assumed that all of the molecules are in the same state, but rather that some are highly polar, some almost non-polar, and others represent all gradations between the two." For Lewis, a non-polar molecule is one "in which the electrons belonging to the individual atom are held by such constraints that they do not move far from their normal positions, while in the polar molecule the electrons, being more mobile, so move as to separate the molecule into positive and negative parts". In the language of quantum mechanics, one could say that there is a probability for a given arrangement of electrons obtained from $|\Psi|^{2}$. In this context one can ask about the relevance of considering only the highest maximum of $|\Psi|^{2}$. For example one can find another maximum corresponding to a structure (figure 3) resembling Lewis' cubical atom[1]. The value of $|\Psi|^{2}$ at this maximum is close to the value at the maximum corresponding to the arrangement shown in figure 2 . Another way to look at this arrangement is that of two displaced tetrahedra, as proposed by Linnett[10] (figure 4).

Also, as $|\Psi|^{2}$ is a probability density, the probability of having the given electron arrange- 
ment can be low if $|\Psi|^{2}$ is sharply peaked around the maximum and the values of $|\Psi|^{2}$ close to the maximum are low. The electron arrangement can be of little significance if there are several other positions yielding also high values of $|\Psi|^{2}$. Thus, instead of searching for the positions of electrons which maximize $|\Psi|^{2}$ it might be more relevant to investigate the regions of space where the probability of finding a pair of electrons is large. We will follow in the present paper this path, searching for the regions of space $\Omega$ where the probability $P_{\Omega}$ to find pairs of electrons of opposite spins is maximal.

Before going on with specific examples, a few remarks:

- We consider here only pairs of electrons with opposite spins as this is assumed to be the quantum mechanical equivalent to Lewis' electron pairs.

- It is natural to find several regions $\Omega$ as they correspond to different bonds or lone pairs.

- We expect to obtain regions which correspond to those where molecular orbitals localize. However, the method we use is not restricted to a given type of wave function. In particular, it is not restricted to single Slater determinants. Within VMC, we have considered in the following explicitly correlated wave functions.

- As we define spatial regions, we define sharp borders. This is to be opposed to localized orbitals which extend over the whole space and are only dominant in some region of space.

- The regions of space we define are not basins as it is done in the theory of Atoms In Molecules[3] or for the Electron Localization Function[11], although we expect some resemblance of our $\Omega \mathrm{s}$ with the latter.

- The different $\Omega$ s we consider may overlap in contrast with the basins above, or with Daudel's loges[12]. The latter correspond to a most favorable partition of space, while we find multiple solutions of our maximization problem. Different approaches to interpret chemical bonding may have different advantages, and may be used with more or less success in a different context. Allowing the domains to overlap may be conceptually less satisfying than partitioning of space (as it also happens with basins), but overlapping may allow, if necessary, to reduce fluctuation of particles between domains. 
- As it is common practice to look at the average number of electrons in a region of space (population), we will also present this number for $\Omega$. It is not an integer, as opposed to the number of electrons associated with the probability used to construct $\Omega$. Note that there is no special reason why the average population and the number of electrons for which the probability has been computed should be identical. However, in practice these two numbers are found to be quite close. Moreover, the population counts also electrons of parallel spins and there are situations where there are more or less than the number of electrons considered when obtaining $\Omega$.

The technical details of our calculations are presented in appendices. The first one presents the calculation of the probabilities in $\Omega$, and the second one presents the algorithm for optimizing the regions of space in order to obtain the $\Omega$ s. The data concerning the geometries, basis functions and Jastrow factors will be provided upon request. Before pursuing with applications, we would like to mention some limitations of our approach.

- The analysis is as good as the wave function we use. VMC allows some flexibility in its choice but the ansatz is also subject to prejudice. In the results presented in this paper, the inclusion of correlation has only little changed the $\Omega$ s and the corresponding probabilities, despite the noticeable separation of the electrons in the maxima of $|\Psi|^{2}$.

- The VMC method performs an exploration of $|\Psi|^{2}$ for a finite number of electron arrangements (configurations). Some information is lost in particular in the regions of low density, as these are rarely explored by VMC. This can give slightly too low populations or slightly too compact domains.

- The convergence criterion of the domain search algorithm may also be a source of error. For instance, if the optimal domain is not completely reached, $\Omega$ can have an overlap region with another domain, which can in some cases be reduced by further optimization.

- $\Omega$ is constructed from a union of small boxes, of size adapted to the number of configurations, which in our case have edges of approximately 0.1 atomic units, which is also typical for other calculations analyzing the chemical bond. In our figures we have chosen not to use interpolation. One one hand, it produces some roughness of surfaces 
shown. However, on the other hand, it gives some visualization of the quality of our calculation.

We would like to mention that some work has already been done for atoms and linear molecules using single determinant wave functions[13-15], as well as correlated wave functions[16]. This paper is the first in which we present non-linear molecules.

\section{APPLICATIONS}

\section{Simple systems}

$\mathrm{CH}_{4}$. Our first example is the methane molecule where the expected results are found: each valence domain contains one proton, and the electron pairs symmetrically partition the valence space (figure 5) in a way which is very similar to that obtained by the topological analysis of ELF displayed in figure 6, and also analogous to domains where orbitals would localize.

Each $\Omega$ is populated by 2.0 electrons. The spin-independent probabilities of finding one, two and three electrons inside an optimal domain corresponding to the $\mathrm{C}-\mathrm{H}$ bond are respectively $0.21,0.55$ and 0.19 (see table I). The spin-dependent probability of finding two electrons with opposite spins is $P(2,1)=0.51$ (where the " 1 " in the notation " $P(2,1)$ " stands for the singlet coupling). For the same population inside the domain, one can compute the probabilities one would obtain if the electrons were independent. The difference of the probabilities with those obtained with independent particles shows the quantum effects. The differences with the probabilities of finding independent electrons (in a statistical sense, by a binomial distribution, and not constructed from a Slater determinant, cf. appendix A) are $-0.06,0.25$ and -0.01 . A clear quantum chemical enhancement of the probability of finding two electrons inside the domain is observed. However, this is not the case for the probabilities of finding one or three electrons. In this latter case, the situation is similar to what would happen with classical particles ignoring the Pauli principle and considered as statistically independent. The probabilities were also calculated in the ELF basin, and all the values are practically equal to those in $\Omega$.

Note that the basins of a topological analysis are defined as a partition of space, so the overlap of the basins is impossible by definition. Here, nothing prevents the domains from 
intersecting, and one can observe a very small overlap between two adjacent domains. This seems to be an artifact of the algorithm, as using a symmetry-based partition of the space yields probabilities which are the same within our accuracy.

$\mathrm{H}_{2} \mathrm{O}$. As in $\mathrm{CH}_{4}$, we obtain a structure with four domains placed at the corners of a tetrahedron with a small overlap. They correspond to the $\mathrm{O}-\mathrm{H}$ bonds and the lone pairs. One of each of these domains is presented in figure 7 . The $\Omega$ s corresponding to lone pairs and bonds are naturally not equivalent. The population in both domains is 2.0, and the probabilities are given in table I as well as the differences of probabilities with the case of independent particles. As for methane, the quantum mechanical enhancement of the probabilities is significant for electron pairs.

$N e$. As spherical symmetry is not imposed in the domain search algorithm, the valence of the neon atom can be described similarly to $\mathrm{CH}_{4}$ and $\mathrm{H}_{2} \mathrm{O}$ by four domains containing each one spin up and one spin down electron. These four domains are equivalent among them, but not unique. Figure 8 shows a domain maximizing the probability of finding two electrons with opposite spins. Each domain contains on average 2.0 electrons, and the probabilities are given in in table $\mathrm{I}$.

The group of four domains can be arbitrarily rotated around the nucleus, in contrast to $\mathrm{CH}_{4}$ and $\mathrm{H}_{2} \mathrm{O}$ where the orientation is fixed by the nuclei. However, in the HF molecule (not shown) the proton defines only one axis, and the three domains representing the lone pairs of the $\mathrm{F}$ atom can freely rotate around it. We can again notice an enhancement of $P(2)$ due to quantum mechanical effects.

As the Electron Localization Function respects by definition the symmetry of the wave function, ELF can see only an average of the "resonant" forms. In cases where there is only one structure, the borders of a domain correspond approximately to the separatrix of the basins of ELF, as in $\mathrm{CH}_{4}$ for example. If there are several equivalent maximum probability domains, ELF will produce an average picture. In the neon atom, the averaging produced by ELF produces a spherical shell, and this one will include both the maximum probability domains as well as their borders. As a result, instead of showing a maximum localization $(\approx 1.0)$, ELF presents a lower value $(\approx 0.8)$.

$N_{2} . \mathrm{N}_{2}$ is a textbook example for the triple bond. Nevertheless, the topological analysis of the Electron Localization Function (ELF) gives a population close to four electrons in the $\mathrm{N} \equiv \mathrm{N}$ bond, which does not correspond to the common Lewis structure[17]. Here, we 
searched for the domains maximizing the probabilities of finding three up and three down electrons between the two nuclei, following the traditional $\sigma / \pi$ interpretation of the bond. The $\Omega$ s obtained are shown in figure 9. The green domain corresponds to the $\sigma$ region of the $\mathrm{N}-\mathrm{N}$ bond, and the yellow domain describes one of the lone pairs. The red domain maximizes the probability of finding three up and three down electrons. The surface has been cut in order to make the $\sigma$ (green) domain visible.

The probabilities are given in table II. For the domain associated to the lone pair, there is a noticeable quantum mechanical enhancement of the probability to find three electrons. This may explain why the ELF basins describing the lone pairs are too populated.

However, there is another solution showing up when searching for an $\Omega$ corresponding to a pair of electrons. This is shown in figure 10. It corresponds to one of the three banana bonds. As for the lone pairs of HF, the group of three domains can freely rotate along the molecular axis.

Notice that there is again another solution for the $\Omega$ s corresponding to electron pairs which correspond to $\pi$ bonds. These can freely rotate around the molecular axis. They are not shown as a figure, but one can imagine their shape by removing the $\sigma$ domain from the $\Omega$ corresponding to the triple bond the and by dividing the remaining part by a plane containing the molecular axis.

$\mathrm{C}_{2} \mathrm{H}_{2}$. The electronic structure of acetylene is similar to the structure of $\mathrm{N}_{2}$. The lone pairs in $\mathrm{N}_{2}$ are replaced by $\mathrm{C}-\mathrm{H}$ bonds in acetylene. Here, one finds domains where the populations agree with the populations of the ELF basins. The corresponding domains are displayed in figure 11. One banana bond domain is presented in figure 12. Due to the cylindrical symmetry, the group of three banana bond domains can freely rotate along the molecular axis. As for $\mathrm{N}_{2}$, one finds $\sigma$ and $\pi$ domains with values of $P(\nu)$ similar to those of the banana bond domains.

The values of the maximal probabilities for the $\mathrm{C}-\mathrm{H}$ and $\mathrm{C} \equiv \mathrm{C}$ bonds (table III) are slightly higher than for the lone pair and for the triple bond of $\mathrm{N}_{2}$. This presents a more localized character of the electrons. If one compares the $\Delta P$ s with those obtained for the domains of $\mathrm{N}_{2}$, the triple bonds in both molecules show the same trend. However, for the domain describing the $\mathrm{C}-\mathrm{H}$ bond, there is no quantum mechanical enhancement of $\Delta P(3)$, as opposed to the domain associated to the lone pair of $\mathrm{N}_{2}$. For the $\mathrm{C}-\mathrm{H}$ domain, there is an enhancement of only one $\Delta P(\nu)$ for each domain, so the ELF partition agrees with the 
$\Omega \mathrm{s}$, as well as the populations.

\section{Overlapping domains}

In the simple systems presented in the previous section, the overlap of the optimal domains, although present, does not seem significant from a chemical point of view. At first sight, one could say that the overlap could result from the noisy character of QMC data, or from the optimization algorithm deforming the domain. However, in some cases, the overlap of the domains is significant, as in the $\mathrm{FHF}^{-}$ion which will be now discussed.

$F F^{-}$. In its ground state, the $\mathrm{FHF}^{-}$ion has an equilibrium geometry of $D_{\infty h}$ symmetry, with an $\mathrm{F}-\mathrm{H}$ bond length equal to $1.12 \AA$ at the $\mathrm{HF} / \mathrm{cc}-\mathrm{pVDZ}$ level. If one tries to find the domain corresponding to an $\mathrm{F}-\mathrm{H}$ bond, one finds the domain displayed in figure 13. This domain contains the proton and a population of 2.0 electrons with the probabilities given in table I. symmetry, the domain corresponding to the other $\mathrm{F}-\mathrm{H}$ bond contains also the proton. In this case, the proton is placed in the domain made of the intersection of the two $\mathrm{F}-\mathrm{H}$ domains. In this particular case, the overlap of the domains can be interpreted as a consequence of the resonance between the $\left[\mathrm{F}-\mathrm{H}+\mathrm{F}^{-}\right]$and $\left[\mathrm{F}^{-}+\mathrm{H}-\mathrm{F}\right]$ structures. In the intersection of the domains, the electron population is 0.1 with $P(0)=0.90$ and $P(1)=0.10$.

A small increase in the $\mathrm{F}-\mathrm{F}$ distance breaks the symmetry and leads to $\left[\mathrm{F}-\mathrm{H}+\mathrm{F}^{-}\right]$ with $\mathrm{F}-\mathrm{H}$ bond lengths equal to $1.08 \AA$ and $1.24 \AA$. In that case, the proton is not located anymore in the intersection (see figure 14). The values for the probabilities and of the $\Delta P \mathrm{~s}$ are the same as for the symmetrical structure up to 0.01. In the intersection of the two $\mathrm{F}-\mathrm{H}$ domains, for both geometries, one finds values of $\Delta P(\nu)$ less than 0.01 , but their sign can give some information. The $\Delta P(0)$ and $\Delta P(2)$ are negative, and the $\Delta P(1)$ are positive. This shows that for both geometries, there is a tiny enhancement of $P(1)$ due to the quantum mechanical interactions of the electrons, and this could explain the existence of the known ELF basin[18] around the proton.

$\mathrm{CH}_{5}^{+}$. Protonated methane is a floppy molecule, which is a challenging problem in rotational-vibrational spectroscopy $[19,20]$. The $\mathrm{CH}_{5}^{+}$ion is, in its ground state, in the $C_{s}$ symmetry. There are other equivalent $C_{s}$ ground states connected by transition states of $C_{2 v}$ symmetry. The activation energy for a rearrangement is very low $(0.8 \mathrm{kcal} / \mathrm{mol})[21]$. 
The chemical bonds cannot be described by a classical Lewis structure since an electron pair is shared by two atoms.

The domains found for the ground state (figure 15) have a structure similar to that of $\mathrm{CH}_{4}$ : four domains maximizing the probability of finding two electrons with opposite spins forming a tetrahedron. Three of the four domains contain one proton, and the other one contains two protons. The latter can be seen as a pair of electrons shared among three centers (three-center two-electron bond), similarly to the picture provided by ELF[22].

The $\mathrm{CH}_{5}^{+}$ion in the transition state is represented in Figure 16 and the probabilities are given in table I. The optimal domains are analogous to those found for the ground state, but one of the protons participating to the three-center bond has moved towards the side of the domain in the intersection with the neighboring domain. Now we have two equivalent domains containing two protons, one proton being shared by these two domains. The description is similar to that of $\mathrm{FHF}^{-}$.

The present description of the bonds agrees with the very fluxional character of the molecule. The structure of the bonding domains does not change when going from the $C_{s}$ to the $C_{2 v}$ conformation. Here, we can see the system as a central carbon nucleus, two core electrons with opposite spins, and five protons moving easily in a fluid of eight valence electrons arranged as four pairs of opposite spins.

\section{Degenerate structures $-\mathrm{Si}_{2} \mathbf{H}_{2}$}

In contrast to $\mathrm{C}_{2} \mathrm{H}_{2}$, the most stable conformation of $\mathrm{Si}_{2} \mathrm{H}_{2}$ has a butterfly structure. However, there exists a local minimum which is closer related to the structure of $\mathrm{C}_{2} \mathrm{H}_{2}$. For a recent study of this trans-bent structure of $\mathrm{Si}_{2} \mathrm{H}_{2}$, see [23-25].

A domain corresponding to the $\mathrm{Si}-\mathrm{H}$ bond was found, with a population of 1.9 electrons. The probabilities are given in table IV. Between the two silicon atoms, we found a domain corresponding to a distorted triple bond populated by 5.8 electrons. The distortion is associated to the push of the $\mathrm{Si}-\mathrm{H}$ bond as shown in figure 17.

Distorted banana bonds of $\mathrm{Si}_{2} \mathrm{H}_{2}$ were also found (figure 18). As opposed to $\mathrm{C}_{2} \mathrm{H}_{2}$, they cannot freely rotate, but there exists only two ways of assigning them, which are related by the inversion symmetry operation. These two structures can be viewed as resonant forms (see bottom of figure 18). The probabilities are given in table IV. 
The optimized domains do not agree with the ELF partition[23], where four equivalent basins with fractional populations are found (figure 19). The two resonant forms may explain the ELF partition. As remarked for $\mathrm{N}_{2}$, ELF shows an average of the resonant forms. The superimposition of the domain corresponding to the "single bond" of one resonant structure and those corresponding to the "double bond" of the other resonant structure decreases the ELF separation between the two domains describing the double bond, so that the double bonds of the upper part of the figure will give raise only to two localization domains, slightly separated by the molecular plane. Close to the plane perpendicular to it containing the silicon nuclei, we can see the borders of maximum probability domains in both structures, and thus expect that the separation in ELF will be more clear.

\section{SUMMARY}

Quantum mechanics requires the delocalization of the electron pairs, leading us to the definition of possibly overlapping domains. The regions maximizing the probability of finding a given number of pairs of electrons with opposite spins were found, and often domains resembling basins of the Electron Localization Function (ELF) or domains of orbital localization were obtained. Simple cases where four electron pairs share the molecular space were described $\left(\mathrm{CH}_{4}, \mathrm{H}_{2} \mathrm{O}, \mathrm{Ne}\right)$. The domains of acetylene are very similar to the ELF basins, and although $\mathrm{N}_{2}$ has an electronic structure very close to that of $\mathrm{C}_{2} \mathrm{H}_{2}$, its domains are slightly different from the ELF basins, and the triple bond can be easily be found with the presented method.

A case which cannot be represented by basins is $\mathrm{FHF}^{-}$where the overlap of the domains is chemically relevant: the proton takes advantage of both $\mathrm{F}-\mathrm{H}$ bonds of the two resonant structures. Another interesting case of overlapping domains is $\mathrm{CH}_{5}^{+}$, where the electronic structure is fixed, and the protons can move easily in the "fluid" made of the four electron pairs.

In some situations, the degeneracy of the optimal domains is self-evident (the four electron pairs of the Neon atom, or the three banana bonds of acetylene). Nevertheless, there exists some structures where this degeneracy is less obvious, as in $\mathrm{Si}_{2} \mathrm{H}_{2}$. 


\section{Appendix A: Calculation of the probabilities in a domain}

Consider an $N$-electron wave function $\Psi$. In a given domain $\Omega$ of the three-dimensional space, the probability of finding $\nu$ electrons inside the domain $\Omega$ and the $N-\nu$ remaining electrons outside of $\Omega$ (inside $\bar{\Omega}$, the complement of $\Omega$ ) is given by (see for example [12]):

$$
P_{\Omega}(\nu)=\frac{N !}{\nu !(N-\nu) !} \int_{\Omega} \mathrm{d} 1 \ldots \mathrm{d} \nu \int_{\bar{\Omega}} \mathrm{d}(\nu+1) \ldots \mathrm{d} N|\Psi|^{2}
$$

The probability can be expressed in terms of spin-dependent probabilities:

$$
P_{\Omega}(\nu)=\sum_{m} P_{\Omega}(\nu, m)
$$

where the number of electrons is $\nu=\nu_{\alpha}+\nu_{\beta}\left(\nu_{\alpha}\right.$ and $\nu_{\beta}$ being respectively the number of spin up and spin down electrons inside the domain) and $m$ is defined as $m=\left|\nu_{\alpha}-\nu_{\beta}\right|+1$. For instance, the probability of finding one anti-parallel pair of electrons and one unpaired electron will be denoted $P_{\Omega}(3,2)$.

Another quantity of interest is $\Delta P_{\Omega}(\nu)$ : the difference between $P_{\Omega}(\nu)$ and the probabilities one would obtain with statistically independent particles, $P_{\Omega \text {,ind }}(\nu)$, considering the population of the domain equal to the population calculated from the $P_{\Omega}(\nu)$.

$$
\Delta P_{\Omega}(\nu)=P_{\Omega}(\nu)-P_{\Omega, \text { ind }}(\nu)
$$

with

$$
P_{\Omega, \text { ind }}(\nu)=\left(\begin{array}{c}
N \\
\nu
\end{array}\right)\left(\frac{\sum_{n=0}^{N} n P_{\Omega}(n)}{N}\right)^{\nu}\left(1-\frac{\sum_{n=0}^{N} n P_{\Omega}(n)}{N}\right)^{N-\nu}
$$

coming from the binomial distribution.

In practice, Variational Quantum Monte Carlo (VMC) simulations are well suited to the problem. VMC consists in the sampling of the $N$-particle density using a random walk with the Metropolis algorithm. Usually, the local energy is averaged in order to calculate the expectation value of the energy associated to the wave function, but here we are interested only in the sampling property of the method. The calculation of all possible probabilities in a given domain is straightforward: at every step of the random walk, one has to count how many up and down electrons are inside the domain, and the spin-dependent probabilities are obtained after building the two-dimensional histogram depending on $\nu$ and $m$.

An important remark can be made: the VMC code is used here only to sample the squared wave function, and not to compute expectation values. One of the major problems 
in usual VMC calculations using low-quality wave functions is the slow decrease of the statistical error on the estimator of the energy as a function of the number of Monte Carlo steps. A lot of work has to be done in order to get accurate energies. For our applications this is not a problem since the squared wave function is sampled whatever the quality of the wave function, and generally a few million configurations are sufficient to get accurate probabilities. This method can thus be applied to large molecules or systems containing atoms with a high nuclear charge. Let us emphasize that any wave function can be sampled within VMC, and therefore the calculation of the probabilities is not restricted to a particular class of wave functions.

\section{Appendix B: Investigation of the domain}

In this appendix, we present the deformation procedure of the domain in order to maximize the probability of finding electron pairs. Here, we assume that a VMC simulation has already been done, and that a large set of independent electron configurations has been saved in a file during the simulation. The probabilities could be calculated on the fly with the VMC code, but the acceleration technique presented at the end of this section imposes us to have the whole set of electron configurations always present in memory.

The user gives to the program a trial domain $\Omega_{0}$, which can be an ELF basin for example. Starting from this initial guess, the deformation of the domain will result from successive local deformations of the surface. The domain is distorted so as to maximize $P_{\Omega}(\nu, m)$ via the function:

$$
f_{\nu, m}(\Omega)=P_{\Omega}(\nu, m) \prod_{(i, j) \neq(\nu, m)}\left(1-P_{\Omega}(i, j)\right)
$$

The function $f_{\nu, m}(\Omega)$ will thus simultaneously maximize $P_{\Omega}(\nu, m)$ and minimize all the $P_{\Omega}(i, j)$ in order to accelerate the convergence towards the maximum of $P_{\Omega}(\nu, m)$.

Now, let us describe the domain deformation. The three-dimensional space is discretized in cells containing Boolean values. The domain $\Omega$ is represented in the grid as the union of all the elementary cells with a value equal to 1 , and the rest of the space is set equal to 0 . At the beginning of the search, $f_{\nu, m}\left(\Omega_{0}\right)$ is calculated for the trial domain and a first iteration of the algorithm starts.

At the beginning of an iteration, all the cells belonging to the surface are identified. The 
list of cells belonging to the surface is explored randomly, in order to avoid a preferred direction of deformation of the domain. For each element of the list of surface cells, one micro-iteration is performed as described in the next paragraph.

The micro-iteration consists in a local deformation of the domain around a cell. First, the current cell $c$ is removed from the domain and $f_{\nu, m}\left(\Omega_{n}^{\prime}\right)$ is calculated $(n$ is the index of the current iteration and $\Omega_{n}^{\prime}$ is the deformed domain). If $f_{\nu, m}\left(\Omega_{n}^{\prime}\right)>f_{\nu, m}\left(\Omega_{n}\right)$, the deformation is favorable for the maximization of $f_{\nu, m}$ and the removal of cell $c$ is accepted. Otherwise, if $f_{\nu, m}\left(\Omega_{n}^{\prime}\right)<f_{\nu, m}\left(\Omega_{n}\right)$ the domain tries to expand around the cell, and there is another step of exploration of all the $3 \times 3 \times 3-1=26$ cells surrounding $c$ which are outside of the domain (with a value equal to zero in the Boolean grid).

In order to avoid a preferred direction for the local growth of the domain, the deformation is made such that its distance to $c$ is constant, in contrast to the algorithm presented in [16]. This not obvious in practice, since the centers of the cells surrounding $c$ are not positioned on a sphere. One way is to explore the cells surrounding $c$, and to select the cells which will be added to the domain with a probabilistic criterion. The Metropolis algorithm was used, where the addition of a cell was accepted with a probability equal to the overlap between the cell and the largest sphere included in the box made of the 27 cells (centered on $c$ ). In this way, the growth of the domain is locally spherical on average. For example, assume we start from an $\Omega_{0}$ which is composed of only one cell, and that one tries to find a domain relative to a core region of an atom. If one selects uniformly all the cells surrounding $c$ at every microiteration, the domain will start to grow as a cube, and will only be deformed as a sphere at the end of the optimization. If the convergence threshold is too low, the optimal domain will not be a perfect sphere. With the probabilistic selection of the cells surrounding $c$, in the given example the domain will always grow spherically. Moreover, this helps to control the homogeneous evolution of the borders of the domain in low density regions. When the cells surrounding $c$ have been processed, $f_{\nu, m}\left(\Omega_{n}^{\prime}\right)$ is calculated. If $f_{\nu, m}\left(\Omega_{n}^{\prime}\right)>f_{\nu, m}\left(\Omega_{n}\right)$, the proposed deformation is favorable for the maximization of $f_{\nu, m}$ and the deformation is accepted. Otherwise, it is rejected.

The calculation of the probabilities is accelerated as follows. Before the first iteration, a list of electron configurations is associated with each one of the elementary cells of the grid. For one cell, the list is composed of all the configurations, read from the file containing the history of the VMC random walks, which have at least one electron inside the cell. In the 
regions of low density, this list is short, and in the regions of high density, this list is usually huge. During a micro-iteration, the values of all the probabilities are updated by taking into account only the configurations present in the list. The update is done as follows. If one cell $c$ has to be removed from $\Omega$, the probabilities are modified such that they take account of all the configurations except those of the list. This is done by calculating, for each configuration of the list, the number of electrons and the spin multiplicity inside $\Omega$, and by subtracting the corresponding value to the two-dimensional histogram introduced in appendix A. Now, the removal of $c$ does not affect the values of the probabilities since none of the set of configurations has an electron inside $c . c$ is then removed from $\Omega$ and we obtain the domain $\Omega^{\prime}$. The probabilities are finally updated such that they consider again all the configurations. This is done by calculating for each configuration which has been removed from the total set (the configurations in the list associated with $c$ ) the number of electrons and the spin multiplicity inside $\Omega^{\prime}$, and by adding the corresponding value to the two-dimensional histogram. The addition of a new cell is done similarly.

When all the micro-iterations have been performed, the resulting domain is regularized with a median filter: each elementary cell is considered at the center of a larger box containing $3 \times 3 \times 3=27$ cells. All the 27 Boolean values are sorted in a list, and the value of the middle element of the list (number 14) is assigned to the cell at the center of the larger box. In other words, if there are more zeros than ones in the larger box, zero is assigned to the central cell. Otherwise, one is assigned to the cell. The purpose of this step is twofold. First, the optimal surface becomes more regular. Second, the number of points belonging to the surface is smaller if the surface is smooth than if the surface is rough: the number of micro-iterations can be considerably reduced, speeding up the calculation. The iteration is now finished. The values of the probabilities are calculated from the two-dimensional histogram, and $f_{\nu, m}\left(\Omega_{n}\right)$ is updated. When $\left(f_{\nu, m}\left(\Omega_{n}\right)-f_{\nu, m}\left(\Omega_{n-1}\right)\right) / f_{\nu, m}\left(\Omega_{n-1}\right)<0.005$, the calculation has converged, otherwise a new iteration is processed.

When the optimal domain is found, $\Omega$ is regularized with a Gaussian filter, where the width of the Gaussian is chosen equal to 0.2 atomic units (roughly twice the step of the grid). By this operation, the original Boolean grid is converted to a grid of real values, and the domain is now blurred. The final border of the domain is chosen as the isosurface of the real grid such that $f_{\nu, m}(\Omega)$ is maximal.

In the calculations performed in this paper, the median length of the list of configurations 
associated with an elementary cell was about 8, with some cells containing empty lists (far away from the molecule) and some cells containing lists of more than $10^{4}$ elements (in the core regions). Hence, one micro-iteration needed about 8 configurations instead of the few millions composing the total set of configurations, thanks to the acceleration procedure presented above. The computer time needed for a domain search was typically between five and fifteen minutes on a regular PC ( 10\% compared to the VMC sampling time), but the memory requirement is the limiting factor: the few million electron configurations are kept in memory for the calculation of the probabilities, and the number of lists to store is about $510^{4}$, with a few lists containing several thousands of elements. To make the storage of the electron configurations more compact, instead of storing three real numbers for the position of an electron we store a pointer to the elementary cell in which it is confined. This reduces the storage by a factor of 6 , which is non-negligible when $1 \mathrm{~Gb}$ of memory is needed to perform a calculation.

The total set of configurations was split in ten smaller sets, after randomizing the full list of configurations to avoid correlations of configurations coming from consecutive steps of the VMC random walk. The probabilities were calculated from the ten sub-lists of configurations, supposed independent among each other, and error bars could then be calculated. In the text, all the values for the probabilities are given with two significant figures. For a fixed domain, the error bars on the probabilities were always below $10^{-2}$. However, the largest error comes from the optimized domain: a small variation of the domain may have little influence on $f_{\nu, m}(\Omega)$, but some effect on the relative values of the probabilities. Hence, as the populations inside the domains are calculated using all the probabilities, they can fluctuate, and we estimate the populations to be significant within 0.1 electrons.

A way to estimate the uncertainty of the border is to perform ten different domain searches from the ten sub-lists of configurations, and to add the different domains to the grid. The cells belonging to $\Omega$ in every optimal domain would have a value of 10 , and the cells always out of $\Omega$ would have a value of 0 . The other cells would represent the uncertainty of the border, with values ranging from 1 to 9 . We started by proceeding in this way, but the optimized domain with ten times less configurations was often much more irregular. 


\section{Appendix C: Computational details}

All the wave functions appearing in this paper were optimized and sampled using the $\mathrm{QMC}=\mathrm{Chem}[26]$ Quantum Monte Carlo program. Trial wave functions were calculated using GAMESS[27] at the Hartree-Fock level using Dunning's cc-pVDZ basis set[28, 29]. Correlation was included using Jastrow factors, optimized by minimizing the variance of the local energy. Generally, 30-70\% of the correlation energy was recovered. The core molecular orbitals were replaced by atomic Slater type orbitals, in order to kill the large core fluctuations, and then to facilitate the optimization of the Jastrow factor. For the special case of $\mathrm{Si}_{2} \mathrm{H}_{2}$, a CAS(10,10)/cc-pVDZ wave function was calculated using GAMESS, and no Jastrow factor was used. A threshold of 0.05 was applied on the coefficients of the spin-adapted configuration functions in order to reduce the number of determinants to 7 . The ELF basins were computed using the TopMod program[30], and all the figures were constructed using the Molekel program[31].

\section{Acknowledgments}

It is our pleasure to dedicate this paper to Professor H. G. von Schnering (Stuttgart) on his 75th birthday for continuously supporting and contributing to the methods helping to understand the chemical bond.

The authors would like to thank the ACI: "Simulation Moléculaire" for financial support, in particular A.Scemama for a post-doctoral position. We also would like to acknowledge interesting discussions with Roland Assaraf (Université Pierre et Marie Curie and CNRS, Paris), Eric Cancès and Tony Lelièvre (both at Ecole Nationale des Ponts et Chaussées, Champs-sur-Marne). We would like to thank Prof. P. Fulde (Dresden) for comments on our manuscript and W.H.E. Schwarz (Siegen) for several suggestions which made us improve it. Finally we would like to thank David L. Cooper (University of Liverpool) for drawing our attention on $\mathrm{Si}_{2} \mathrm{H}_{2}$ and Gernot Frenking (Philipps-Universität Marburg) for fruitful discussions concerning this molecule.

Some of the numerical calculations have been performed using the computational resources of IDRIS (CNRS, Orsay) and CALMIP (Toulouse). 
[1] Lewis, G. N. J. Am. Chem. Soc. 1916, 38, 762.

[2] Langmuir, I. J. Am. Chem. Soc. 1919, 41, 868.

[3] Bader, R. F. W. Atoms in Molecules: A Quantum Theory 1990, Clarendon, Oxford.

[4] Lennard-Jones, J. E. J. Chem. Phys. 1952, 20, 1024.

[5] Becke, A. D.; Edgecombe, K. E. J. Chem. Phys. 1990, 92, 5397.

[6] Mulliken, R. S. J. Chem. Phys. 1955, 23, 1833-1840, 1841-1846, 2338-2342, 2343-2346.

[7] Artmann, K. Z. Naturforsch. 1946, 1, 426.

[8] Zimmermann, H. K.; Van Rysselberghe,P. J. Chem. Phys. 1949, 17, 598.

[9] Fulde P., "Electron Correlations in Molecules and Solids" Springer Series in Solid State Sciences Vol. 100, 3rd ed. 1995.

[10] Linnett, J. W. J. Am. Chem. Soc. 1961, 83, 2643.

[11] Silvi, B.; Savin, A. Nature 1994, 371, 683.

[12] Aslangul, C.; Constanciel, R.; Daudel, R.; Kottis, P. Adv. Quant. Chem. 1972, 6, 93.

[13] Gallegos, A.; Carbó-Dorca, R.; Lodier, F.; Cancès, E.; Savin, A. J. Comp. Chem 2005, 26, 455.

[14] Cancès, E.; Keriven, R.; Lodier, F.; Savin, A. Theor. Chem. Acc. 2004, 111, 373.

[15] Savin, A. Reviews of modern quantum chemistry: A celebration of the contributions of Robert G. Parr; K. D. Sen Ed.; World Scientific: Singapore, 2002; 43.

[16] Scemama, A. J. Theor. Comp. Chem. 2005, 4, 397.

[17] Ponec, R.; Chaves, J. J. Comp. Chem. 2006, this issue.

[18] Savin, A.; Becke, A. D.; Flad, J.; Nesper, R.; Preuss, H.; Von Schnering, H. G. Angew. Chem. Int. Ed. Engl. 1991, 30, 409.

[19] Marx, D.; Parrinello, M. Science 1999, 284, 59.

[20] Asvany, O.; Kumar P, P.; Redlich, B.; Hegemann, I.; Schlemmer, S.; Marx, D. Science 2005, 309, 1219-1222.

[21] Müller, H.; Kutzelnigg, W.; Noga, J.; Klopper, W. J. Chem. Phys. 1997, 106, 1863.

[22] Marx, D.; Savin, A. Angew. Chem. Int. Ed. Engl. 1997, 36, 2077.

[23] Silvi, B; Fourré, I.; Alikhani, M. E. Monatshefte für Chemie 2005, 136, 855.

[24] Lein, M.; Krapp, A.; Frenking, G. J. Am. Chem. Soc. 2005, 127, 6290. 
[25] Danovich, D.; Ogliaro, F.; Karni, M.; Apeloig, Y.; Cooper, D. L.; Shaik, S. Angew. Chem. Int. Ed. 2001, 40, 4023.

[26] $\mathrm{QMC}=$ Chem is a Quantum Monte Carlo program written by M. Caffarel, IRSAMC, Université Paul Sabatier - CNRS, Toulouse, France.

[27] Schmidt, M. W.; Baldridge, K. K.; Boatz, J. A.; Elbert, S. T.; Gordon, M. S.; Jensen, J. H.; Koseki, S.; Matsunaga, N.; Nguyen, K. A.; Su, S. J.; Windus, T. L.; Dupuis, M.; Montgomery. J. A. J. Comp. Chem. 1993, 14, 1347-1363.

[28] Dunning Jr., T.H. J. Chem. Phys. 1989, 90, 1007, and

[29] Woon, D.E.; Dunning Jr., T.H. J. Chem. Phys. 1993, 98, 1358.

[30] $\mathrm{QMC}=$ Chem is a Quantum Monte Carlo program written by Noury, S.; Krokidis, X.; Fuster, F.; Silvi, Université Pierre et Marie Curie, Paris, France.

[31] Portmann, S.; Lüthi, H. P. Chimia 2000, 54, 766. 


\section{Table captions}

- Table I: Spin-independent probabilities $P(\nu)$ of finding $\nu$ electrons, spin-dependent probability $P(2,1)$ of finding one opposite-spin electron pair, and population in each one of the domains $\Omega$ of $\mathrm{CH}_{4}, \mathrm{H}_{2} \mathrm{O}, \mathrm{Ne}, \mathrm{FHF}^{-}$in and out of equilibrium, the $C_{s}$ and $C_{2 v}$ structures of $\mathrm{CH}_{5}^{+} \cdot \Delta P(\nu)$, the differences with probabilities in the case of statistically independent particles, are given in brackets. All domains maximize $P(2,1)$.

- Table II: Spin-independent probabilities $P(\nu)$ of finding $\nu$ electrons, spin-dependent probability $P(2,1)$ of finding one opposite-spin electron pair, and population in each one of the domains $\Omega$ of $\mathrm{N}_{2} \cdot \Delta P(\nu)$, the differences with probabilities in the case of statistically independent particles, are given in brackets. The lone pair domain and the $\sigma$ bond domain maximize $P(2,1)$ and the triple bond domain maximizes $P(6,1)$, the spin-dependent probability of finding three up electrons and three down electrons. The banana bond domain maximizes $P(2,1)$.

- Table III: Spin-independent probabilities $P(\nu)$ of finding $\nu$ electrons, spin-dependent probability $P(2,1)$ of finding one opposite-spin electron pair, and population in each one of the domains $\Omega$ of $\mathrm{C}_{2} \mathrm{H}_{2} . \Delta P(\nu)$, the differences with probabilities in the case of statistically independent particles, are given in brackets. The lone pair domain and the $\sigma$ bond domain maximize $P(2,1)$ and the triple bond domain maximizes $P(6,1)$, the spin-dependent probability of finding three up electrons and three down electrons. The banana bond domain maximizes $P(2,1)$.

- Table IV: Spin-independent probabilities $P(\nu)$ of finding $\nu$ electrons, spin-dependent probability $P(2,1)$ of finding one opposite-spin electron pair, and population in each one of the domains $\Omega$ of $\mathrm{Si}_{2} \mathrm{H}_{2} . \Delta P(\nu)$, the differences with probabilities in the case of statistically independent particles, are given in brackets. The $\mathrm{Si}-\mathrm{H}$ bond and the banana bond domains maximize $P(2,1)$. The triple bond domain maximized $P(6,1)$. 
Tables 


\begin{tabular}{lccccccc}
\hline & $P(0)$ & $P(1)$ & $P(2)$ & $P(3)$ & $P(4)$ & $P(2,1)$ & Pop. \\
\hline \hline $\mathrm{C}-\mathrm{H}$ bond & 0.02 & 0.21 & 0.55 & 0.19 & 0.03 & 0.51 & 2.0 \\
in $\mathrm{CH}_{4}$ & $(-0.09)$ & $(-0.06)$ & $(0.25)$ & $(-0.01)$ & $(-0.06)$ & & \\
\hline \hline O-H bond & 0.04 & 0.26 & 0.46 & 0.20 & 0.04 & 0.39 & 1.9 \\
in $\mathrm{H}_{2} \mathrm{O}$ & $(-0.07)$ & $(-0.02)$ & $(0.15)$ & $(0.00)$ & $(-0.04)$ & & \\
\hline Lone pair & 0.05 & 0.26 & 0.42 & 0.21 & 0.05 & 0.39 & 2.0 \\
in $\mathrm{H}_{2} \mathrm{O}$ & $(-0.06)$ & $(-0.01)$ & $(0.12)$ & $(0.01)$ & $(-0.04)$ & & \\
\hline \hline Lone pair & 0.06 & 0.26 & 0.39 & 0.22 & 0.06 & 0.30 & 2.0 \\
in Ne & $(-0.05)$ & $(-0.01)$ & $(0.09)$ & $(0.02)$ & $(-0.03)$ & & \\
\hline \hline F-H bond & 0.06 & 0.27 & 0.39 & 0.21 & 0.06 & 0.30 & 2.0 \\
in FHF & $(-0.07)$ & $(0.00)$ & $(0.11)$ & $(0.02)$ & $(-0.03)$ & & \\
\hline \hline $\begin{array}{l}\text { Three-center bond in } \mathrm{CH}_{5}^{+} \\
0.02\end{array}$ & 0.22 & 0.52 & 0.20 & 0.03 & 0.47 & 2.0 \\
$C_{s}$ symmetry & $(-0.08)$ & $(-0.05)$ & $(0.22)$ & $(0.00)$ & $(-0.06)$ & & \\
\hline $\begin{array}{l}\text { Three-center bond in } \mathrm{CH}_{5}^{+} \\
0.03\end{array}$ & $\begin{array}{r}0.24 \\
C_{2 v} \text { symmetry }\end{array}$ & 0.54 & 0.16 & 0.02 & 0.50 & 1.9 \\
\hline \hline
\end{tabular}

TABLE I: 


\begin{tabular}{|c|c|c|c|c|c|c|c|c|c|c|c|}
\hline & $P(0)$ & $P(1)$ & $P(2)$ & $P(3)$ & $P(4)$ & $P(5)$ & $P(6)$ & $P(7)$ & $P(8)$ & $P(2,1)$ & Pop \\
\hline \multirow[t]{2}{*}{ Lone Pair } & 0.04 & 0.25 & 0.46 & 0.20 & 0.04 & 0.00 & & & & 0.40 & 2.0 \\
\hline & $(-0.01)$ & $(-0.02)$ & $(0.17)$ & $(0.12)$ & $(-0.05)$ & $(-0.02)$ & & & & & \\
\hline \multirow[t]{2}{*}{$\sigma$ bond } & 0.07 & 0.28 & 0.39 & 0.20 & 0.05 & 0.01 & & & & 0.29 & 1.9 \\
\hline & $(-0.06)$ & $(0.00)$ & $(0.09)$ & $(0.02)$ & $(-0.03)$ & $(-0.02)$ & & & & & \\
\hline \multirow[t]{2}{*}{$\pi$ bond } & 0.07 & 0.28 & 0.37 & 0.21 & 0.06 & 0.01 & & & & 0.28 & 2.0 \\
\hline & $(-0.05)$ & $(0.00)$ & $(0.08)$ & $(0.02)$ & $(-0.02)$ & $(-0.02)$ & & & & & \\
\hline \multirow[t]{2}{*}{ Banana bond } & 0.06 & 0.27 & 0.39 & 0.21 & 0.06 & 0.01 & & & & 0.29 & 2.0 \\
\hline & $(-0.06)$ & $(0.00)$ & $(0.10)$ & $(0.02)$ & $(-0.03)$ & $(-0.02)$ & & & & & \\
\hline \multirow[t]{2}{*}{ Triple bond } & & & 0.00 & 0.03 & 0.11 & 0.24 & 0.31 & 0.21 & 0.08 & & 5.9 \\
\hline & & & $(-0.02)$ & $(-0.04)$ & $(-0.03)$ & $(0.05)$ & $(0.09)$ & $(0.04)$ & $(-0.03)$ & & \\
\hline
\end{tabular}

TABLE II: 


\begin{tabular}{|c|c|c|c|c|c|c|c|c|c|c|c|}
\hline & $P(0)$ & $P(1)$ & $P(2)$ & $P(3)$ & $P(4)$ & $P(5)$ & $P(6)$ & $P(7)$ & $P(8)$ & $P(2,1)$ & Pop. \\
\hline \multirow[t]{2}{*}{$\mathrm{C}-\mathrm{H}$ bond } & 0.02 & 0.20 & 0.57 & 0.19 & 0.03 & 0.00 & & & & 0.53 & 2.0 \\
\hline & $(-0.10)$ & $(-0.07)$ & $(0.27)$ & $(-0.01)$ & $(-0.06)$ & $(-0.03)$ & & & & & \\
\hline \multirow[t]{2}{*}{$\sigma$ bond } & 0.06 & 0.27 & 0.38 & 0.22 & 0.06 & 0.01 & & & & 0.27 & 2.0 \\
\hline & $(-0.05)$ & $(-0.05)$ & $(0.08)$ & $(0.03)$ & $(-0.02)$ & $(-0.02)$ & & & & & \\
\hline \multirow[t]{2}{*}{$\pi$ bond } & 0.06 & 0.27 & 0.38 & 0.21 & 0.06 & 0.01 & & & & 0.28 & 2.0 \\
\hline & $(-0.06)$ & $(0.00)$ & $(0.09)$ & $(0.03)$ & $(-0.03)$ & $(-0.02)$ & & & & & \\
\hline \multirow[t]{2}{*}{ Banana bond } & 0.05 & 0.26 & 0.40 & 0.22 & 0.06 & 0.01 & & & & 0.31 & 2.0 \\
\hline & $(-0.06)$ & $(-0.08)$ & $(0.11)$ & $(0.02)$ & $(-0.03)$ & $(-0.02)$ & & & & & \\
\hline \multirow[t]{2}{*}{ Triple bond } & & & 0.00 & 0.01 & 0.07 & 0.23 & 0.36 & 0.23 & 0.07 & & 6.0 \\
\hline & & & $(-0.02)$ & $(-0.05)$ & $(-0.05)$ & $(0.04)$ & $(0.15)$ & $(0.05)$ & $(-0.04)$ & & \\
\hline
\end{tabular}

TABLE III: 


\begin{tabular}{|c|c|c|c|c|c|c|c|c|c|c|c|}
\hline & $P(0)$ & $P(1)$ & $P(2)$ & $P(3)$ & $P(4)$ & $P(5)$ & $P(6)$ & $P(7)$ & $P(8)$ & $P(2,1)$ & Pop. \\
\hline \multirow[t]{2}{*}{$\mathrm{Si}-\mathrm{H}$ bond } & 0.02 & 0.22 & 0.59 & 0.15 & 0.02 & & & & & 0.56 & 1.9 \\
\hline & $(-0.11)$ & $(-0.06)$ & $(0.30)$ & $(-0.03)$ & $(-0.06)$ & & & & & & \\
\hline \multirow[t]{2}{*}{ Banana bond } & 0.07 & 0.27 & 0.38 & 0.21 & 0.06 & 0.01 & & & & 0.28 & 2.0 \\
\hline & $(-0.06)$ & $(0.02)$ & $(0.10)$ & $(0.02)$ & $(-0.02)$ & $(-0.02)$ & & & & & \\
\hline \multirow[t]{2}{*}{ Two Equivalent Banana bonds } & 0.07 & 0.28 & 0.38 & 0.20 & 0.05 & 0.01 & & & & 0.26 & 1.9 \\
\hline & $(-0.06)$ & $(0.01)$ & $(0.11)$ & $(0.04)$ & $(-0.03)$ & $(-0.02)$ & & & & & \\
\hline \multirow[t]{2}{*}{ Triple bond } & & & 0.01 & 0.03 & 0.12 & 0.25 & 0.29 & 0.19 & 0.08 & & 5.8 \\
\hline & & & $(-0.03)$ & $(-0.05)$ & $(-0.02)$ & $(0.07)$ & $(0.11)$ & $(0.04)$ & $(-0.02)$ & & \\
\hline
\end{tabular}

TABLE IV: 


\section{Figure captions}

- Fig 1: Positions of the electron pairs at a maximum of the squared single determinant wave function, in the water molecule. The positions of the electron pairs (one spin up and one spin down at the same position) are shown as blue spheres. The locations of the hydrogen nuclei are represented as transparent white spheres, and that of the oxygen nucleus as a transparent red sphere. The electron pairs are connected with sticks to show the tetrahedral configuration.

- Fig 2: Positions of the electron pairs at a maximum of the squared correlated wave function, in the water molecule. The positions of the spin up electrons are shown as blue spheres and those of the spin down electrons are shown as green spheres. The location of the hydrogen nuclei are represented as transparent white spheres, and that of the oxygen nucleus as a transparent red sphere. Sticks join the electrons of a pair.

- Fig 3: Positions of the electron pairs at another maximum of the squared correlated wave function in the water molecule. The positions of the spin up electrons are shown as blue spheres and those of the spin down electrons are shown as green spheres. The location of the hydrogen nuclei are represented as transparent white spheres, and that of the oxygen nucleus as a transparent red sphere. Sticks join the electrons of a pair, and a cube has been added to the figure to show that the electron pairs almost form Lewis' cubical atom[1].

- Fig 4: Positions of the electron pairs at the same maximum as in figure 3 of the squared correlated wave function in the water molecule. The positions of the spin up electrons are shown as blue spheres, those of the spin down electrons are shown as green spheres, those of the hydrogen nuclei as transparent white spheres, and that of the oxygen nucleus as a transparent red sphere. The spin up and spin down electrons are both connected together with sticks. Remark that the electrons of same spin are placed at the vertices of a tetrahedron, as suggested by Linnett[10].

- Fig 5: A domain maximizing the probability of finding two electrons with opposite spins, in $\mathrm{CH}_{4}$.

- Fig 6: A basin of the Electron Localization Function corresponding to a $\mathrm{C}-\mathrm{H}$ bond in 
$\mathrm{CH}_{4}$.

- Fig 7: Domains maximizing the probability of finding two electrons with opposite spins, in $\mathrm{H}_{2} \mathrm{O}$. The yellow domain represents the lone pair domain and the transparent blue domain corresponds to the $\mathrm{O}-\mathrm{H}$ bond.

- Fig 8: A domain maximizing the probability of finding two electrons with opposite spins (yellow), in the neon atom. The position of the nucleus is represented by a red sphere.

- Fig 9: Domains maximizing the probability of finding one (yellow and green), and three (red) anti-parallel electron pairs (three spins up and three spins down) in $\mathrm{N}_{2}$. The red domain was made transparent to show the positions of the two nuclei (blue spheres). One can distinguish here the $\sigma$ and $\pi$ regions of the $\mathrm{N}-\mathrm{N}$ bond.

- Fig 10: One banana bond domain (green) of $\mathrm{N}_{2}$ : a domain maximizing the probability of finding two electrons with opposite spins. The surface delimiting the domain relative to the triple bond (red) has been cut to show that the banana bond is included in it.

- Fig 11: Domains maximizing the probability of finding one (transparent white and green) and three (red) anti-parallel electron pairs (three spins up and three spins down) in $\mathrm{C}_{2} \mathrm{H}_{2}$. The red domain was cut to make the green domain visible, as well as the positions of the carbon nuclei (gray spheres). The white spheres represent the positions of the protons.

- Fig 12: One banana bond domain (green) of $\mathrm{C}_{2} \mathrm{H}_{2}$ : a domain maximizing the probability of finding two electrons with opposite spins. The surface delimiting the domain relative to the triple bond (red) has been cut to show that the banana bond is included in it. The positions of the nuclei are shown as spheres.

- Fig 13: Domains corresponding to the $\mathrm{F}-\mathrm{H}$ bonds in $\mathrm{FHF}^{-}$at the equilibrium geometry. The positions of the fluorine nuclei are represented by gray spheres. The proton, represented by a white sphere, is contained in both domains.

- Fig 14: Domains corresponding to the $\mathrm{F}-\mathrm{H}$ bonds in $\mathrm{FHF}^{-}$out of equilibrium. The positions of the fluorine nuclei are represented by gray spheres. The proton, represented by a white sphere, is contained only in the white domain. 
- Fig 15: $\mathrm{CH}_{5}^{+}$in its ground state. The domain corresponding to the three-center bond is shown with dots. A domain relative to a $\mathrm{C}-\mathrm{H}$ bond is displayed in order to show the overlap between the two domains.

- Fig 16: $\mathrm{CH}_{5}^{+}$in the transition state. The proton is contained in the intersection of the transparent and the dotted domains.

- Fig 17: Trans-bent structure of $\mathrm{Si}_{2} \mathrm{H}_{2}$ : The optimal domains correspond to the $\mathrm{Si}-\mathrm{H}$ bond (white) and a distorted $\mathrm{Si}-\mathrm{Si}$ triple bond (blue). The domains have been cut along the molecular plane to show the distorted character of the triple bond domain.

- Fig 18: Banana bonds of $\mathrm{Si}_{2} \mathrm{H}_{2}$. The domains were cut in a plane orthogonal to the $\mathrm{Si}-\mathrm{Si}$ axis in order to show their overlap. The two resonant structures are displayed at the bottom of the figure, and the domains displayed correspond to the resonant structure on the left.

- Fig 19: Isosurfaces of the Electron Localization Function in the trans-bent structure of $\mathrm{Si}_{2} \mathrm{H}_{2}$. The isosurface $\mathrm{ELF}=0.88$ is displayed in transparent yellow, and the isosurface $\mathrm{ELF}=0.94$ (in full yellow) shows the positions of the maxima. 
Figures 


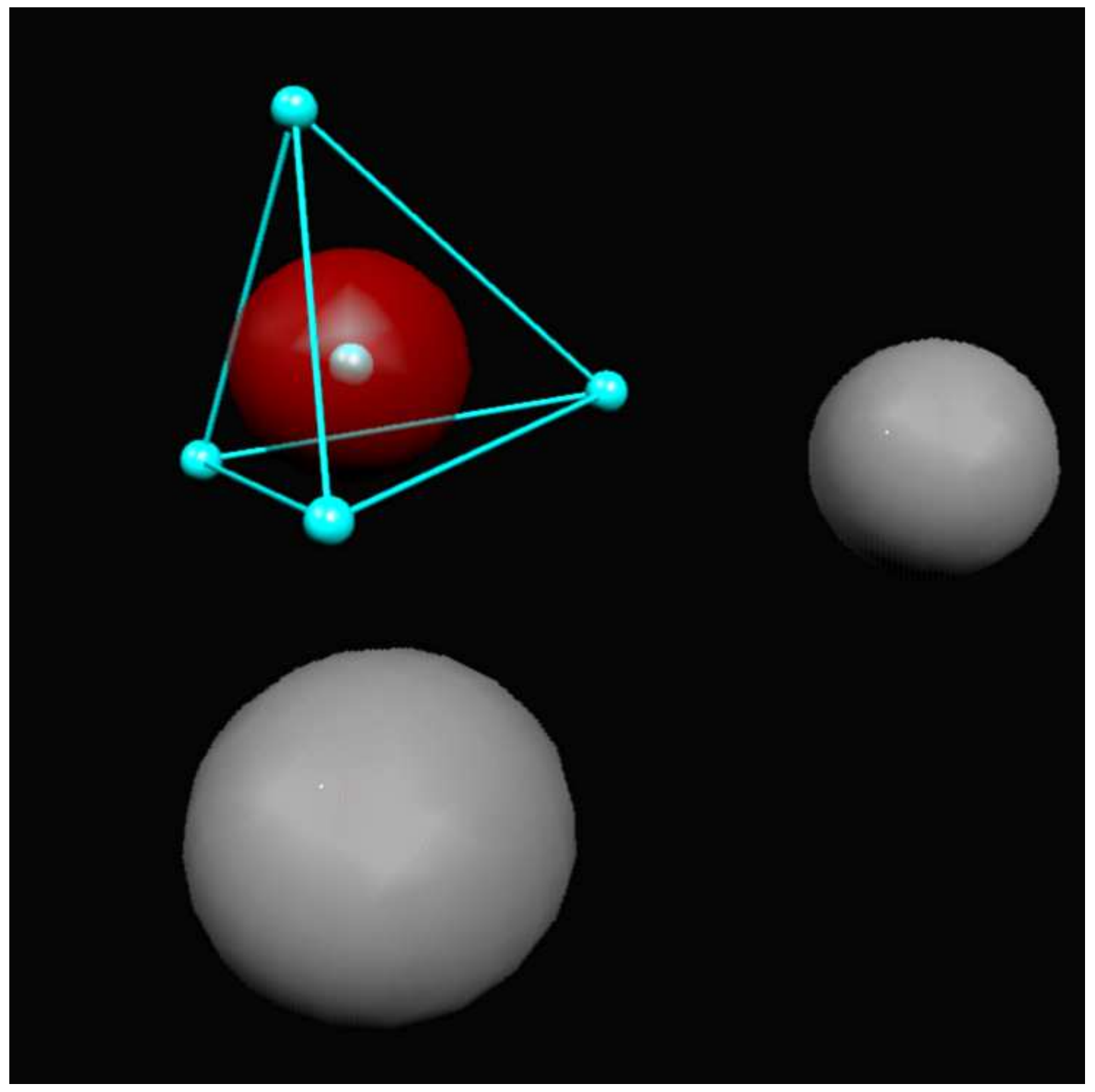

FIG. 1: 


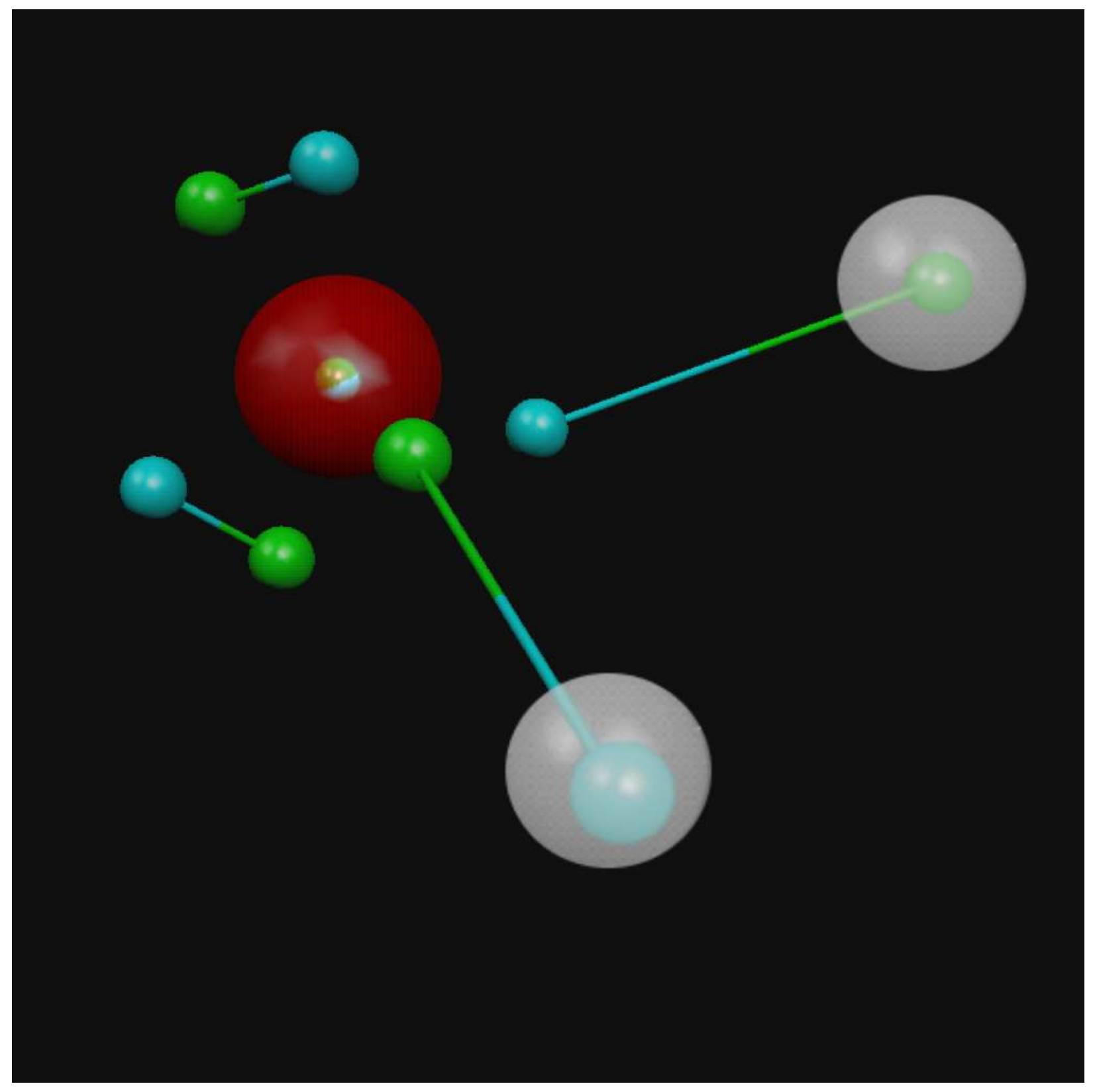

FIG. 2: 


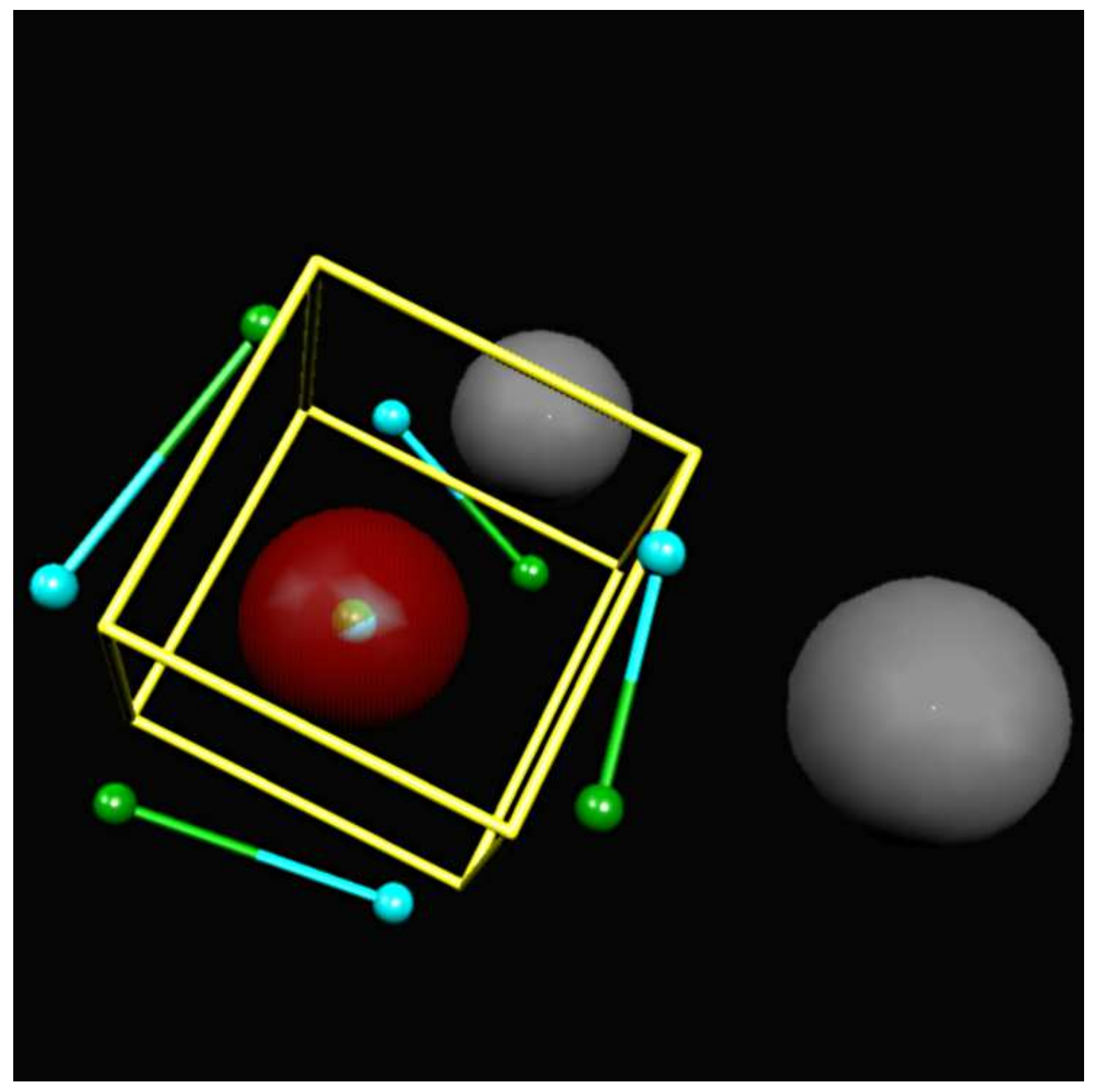

FIG. 3: 


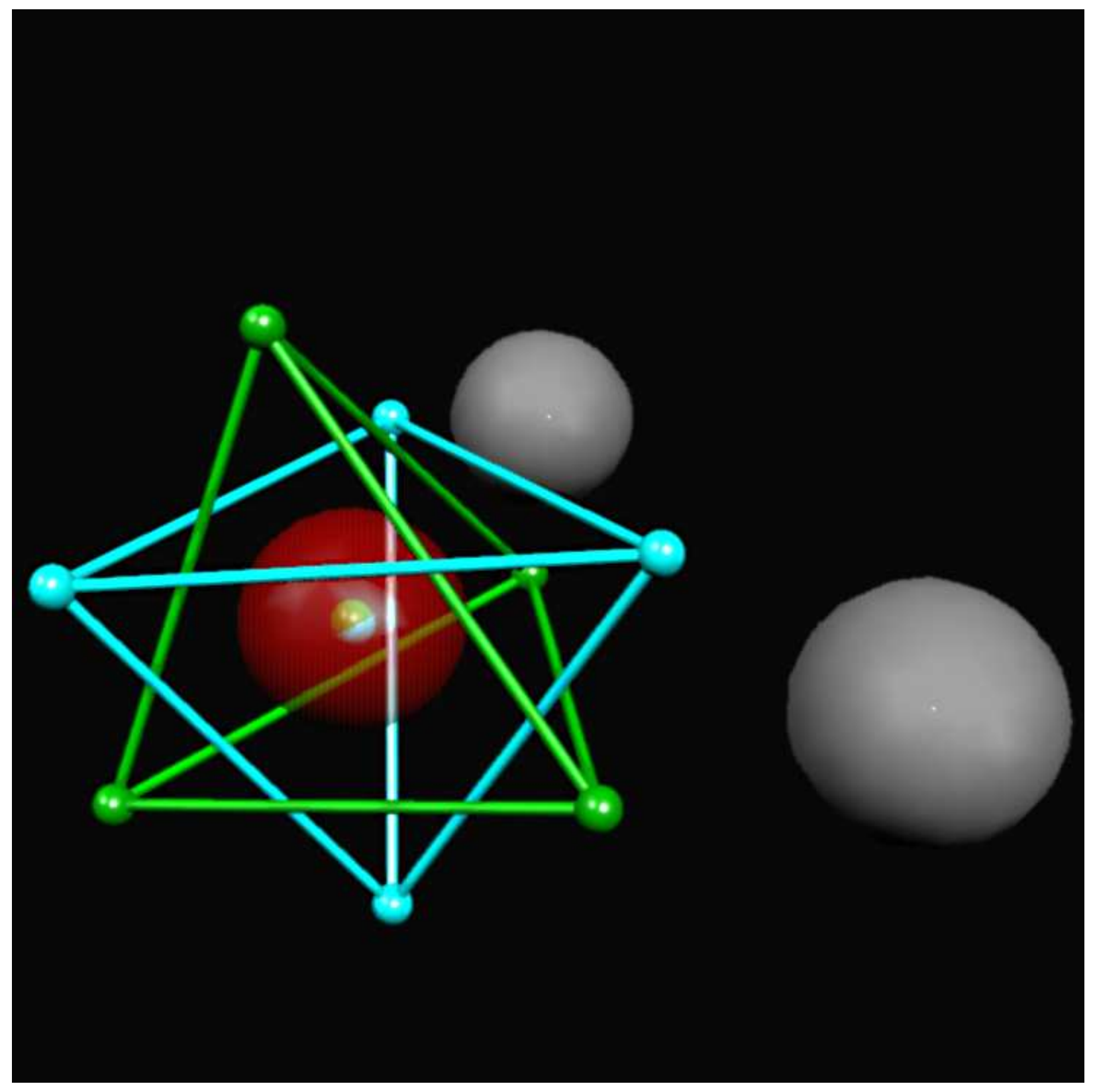

FIG. 4: 


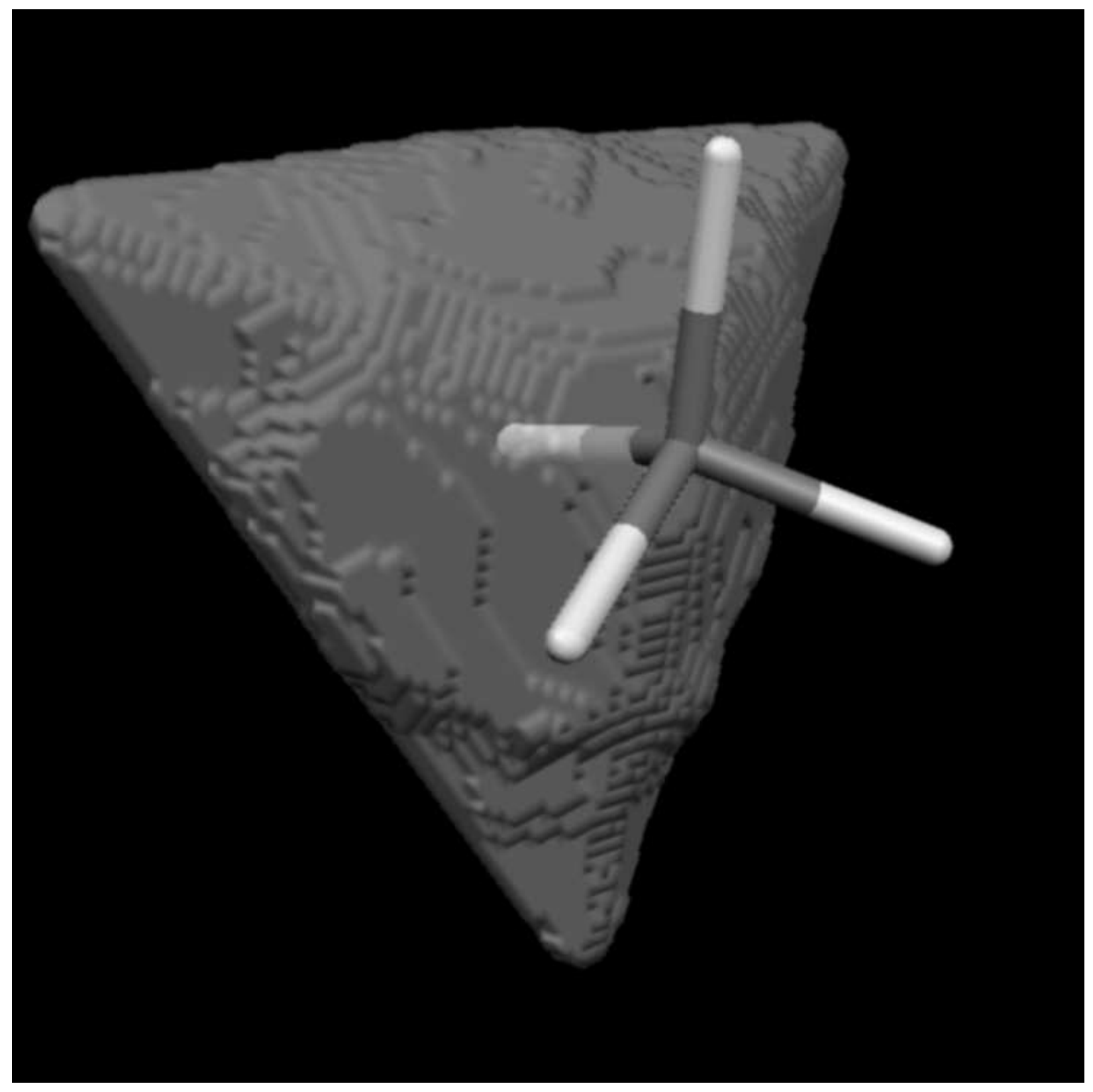

FIG. 5: 


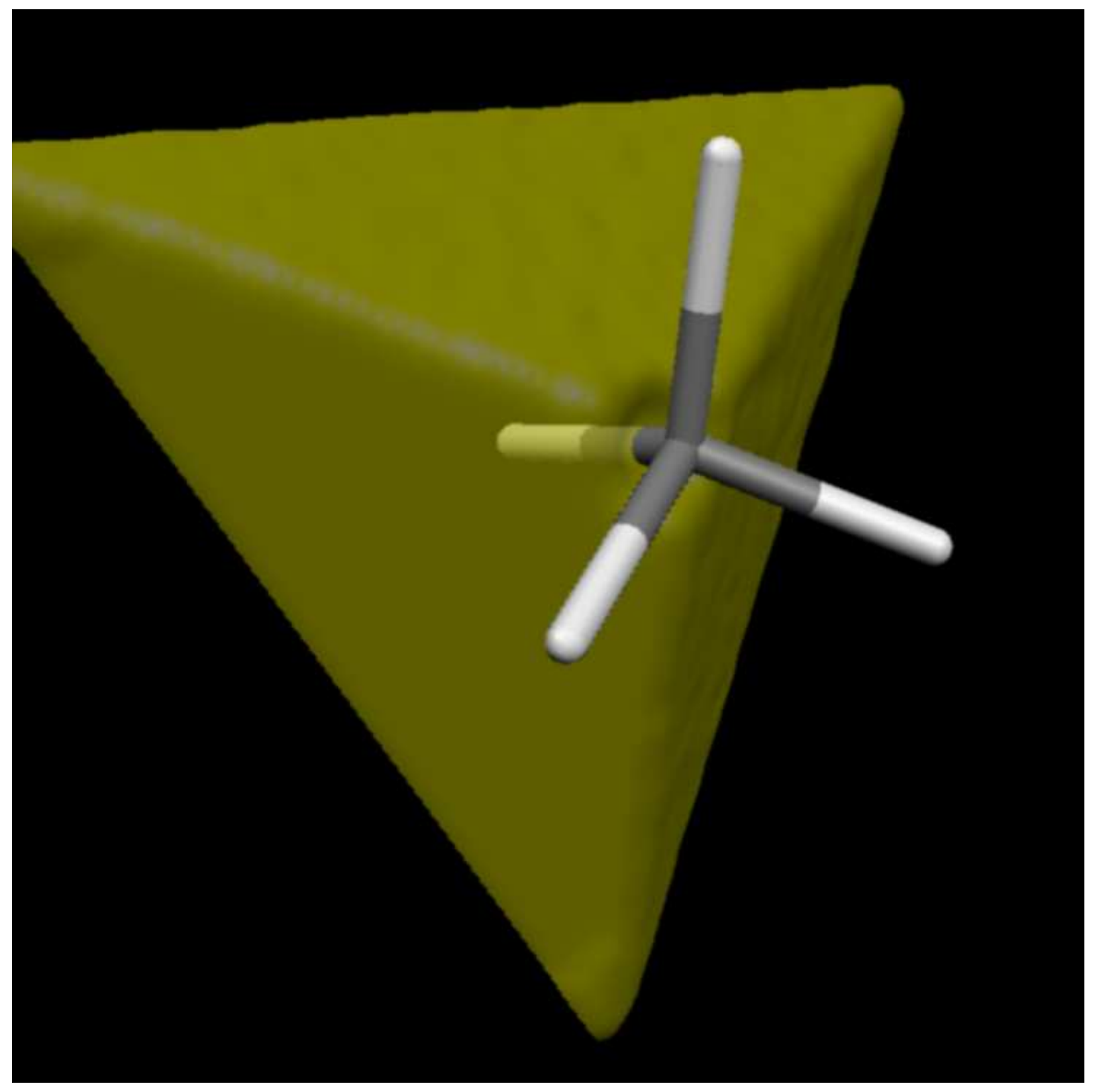

FIG. 6: 


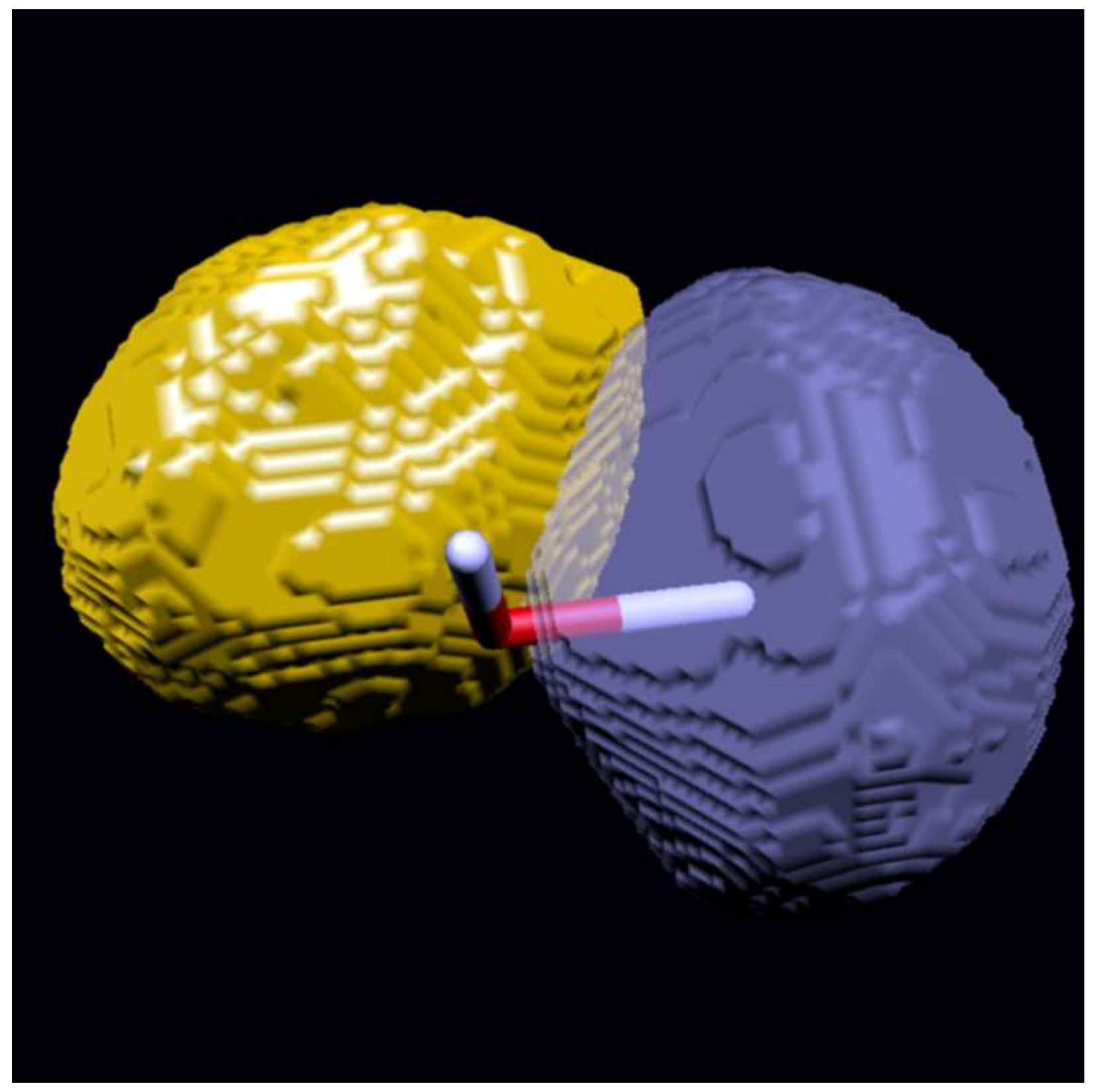

FIG. 7: 


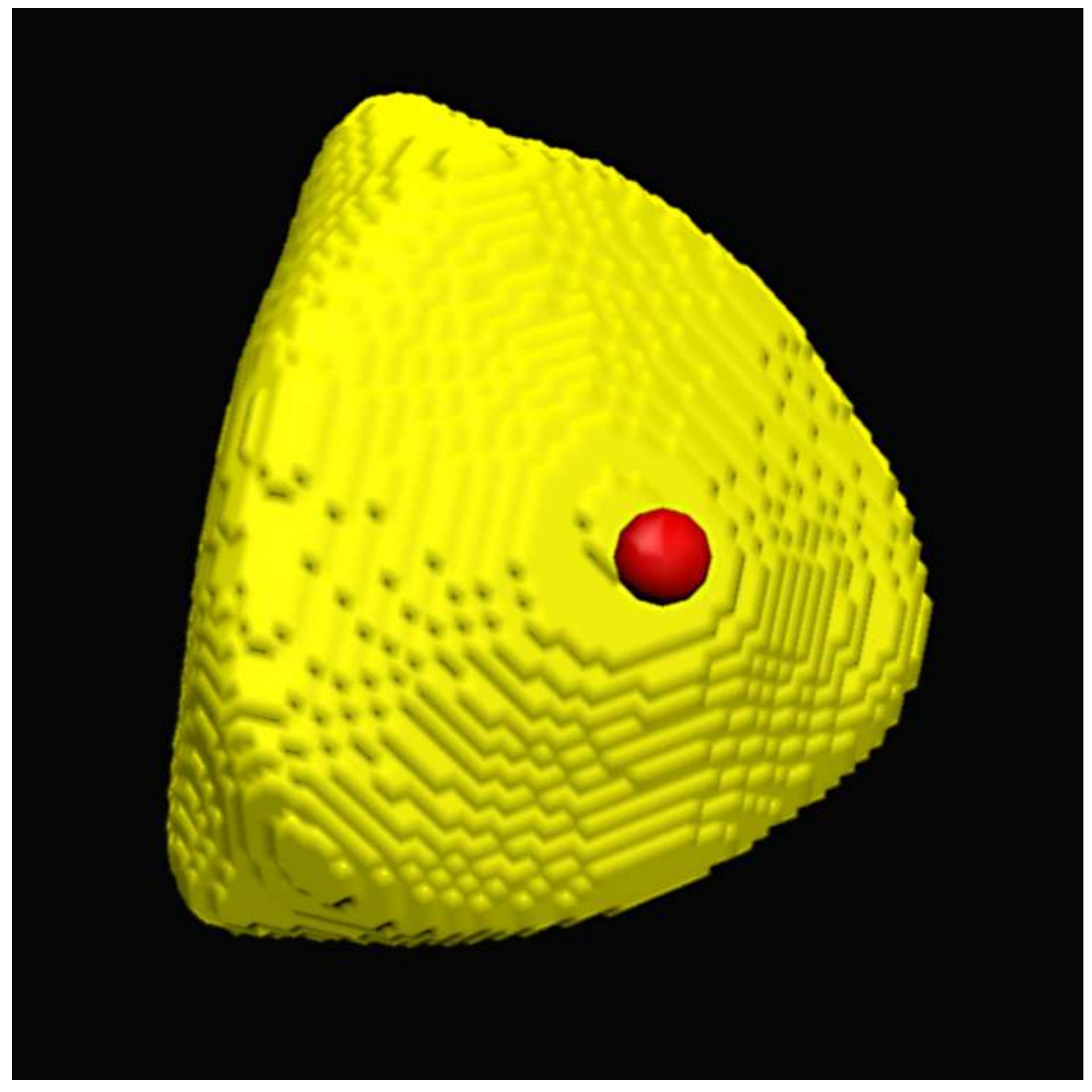

FIG. 8: 


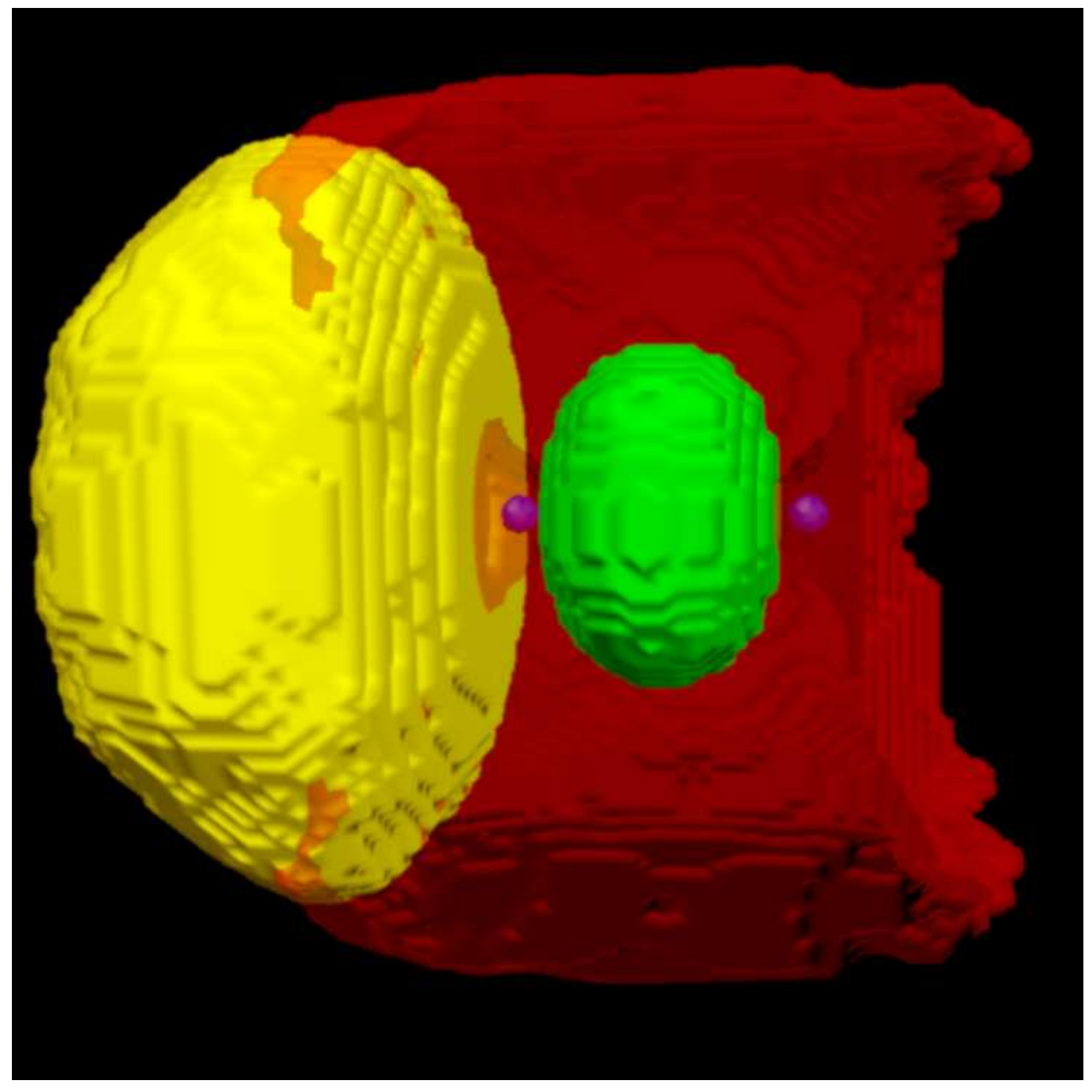

FIG. 9: 


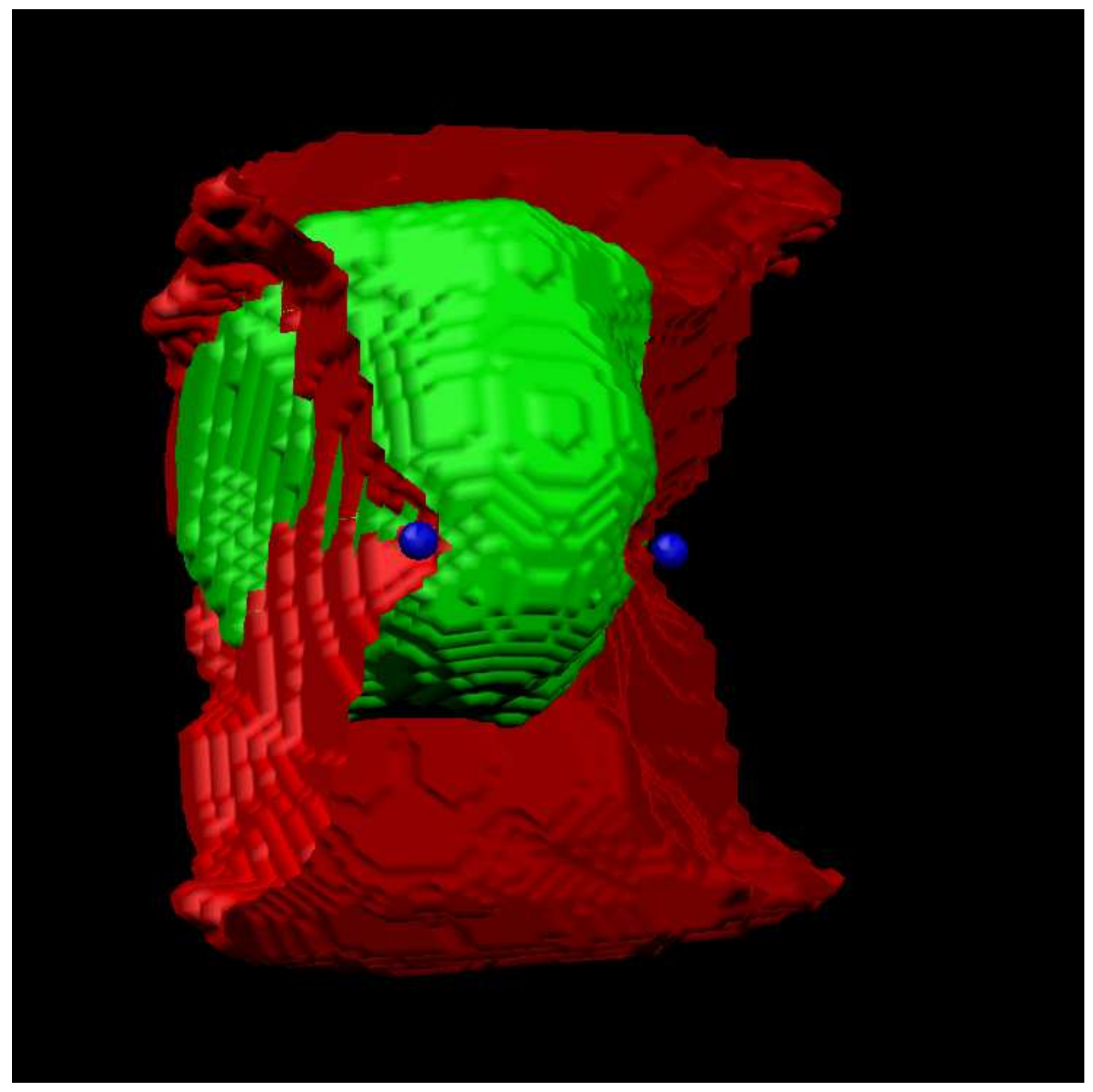

FIG. 10: 


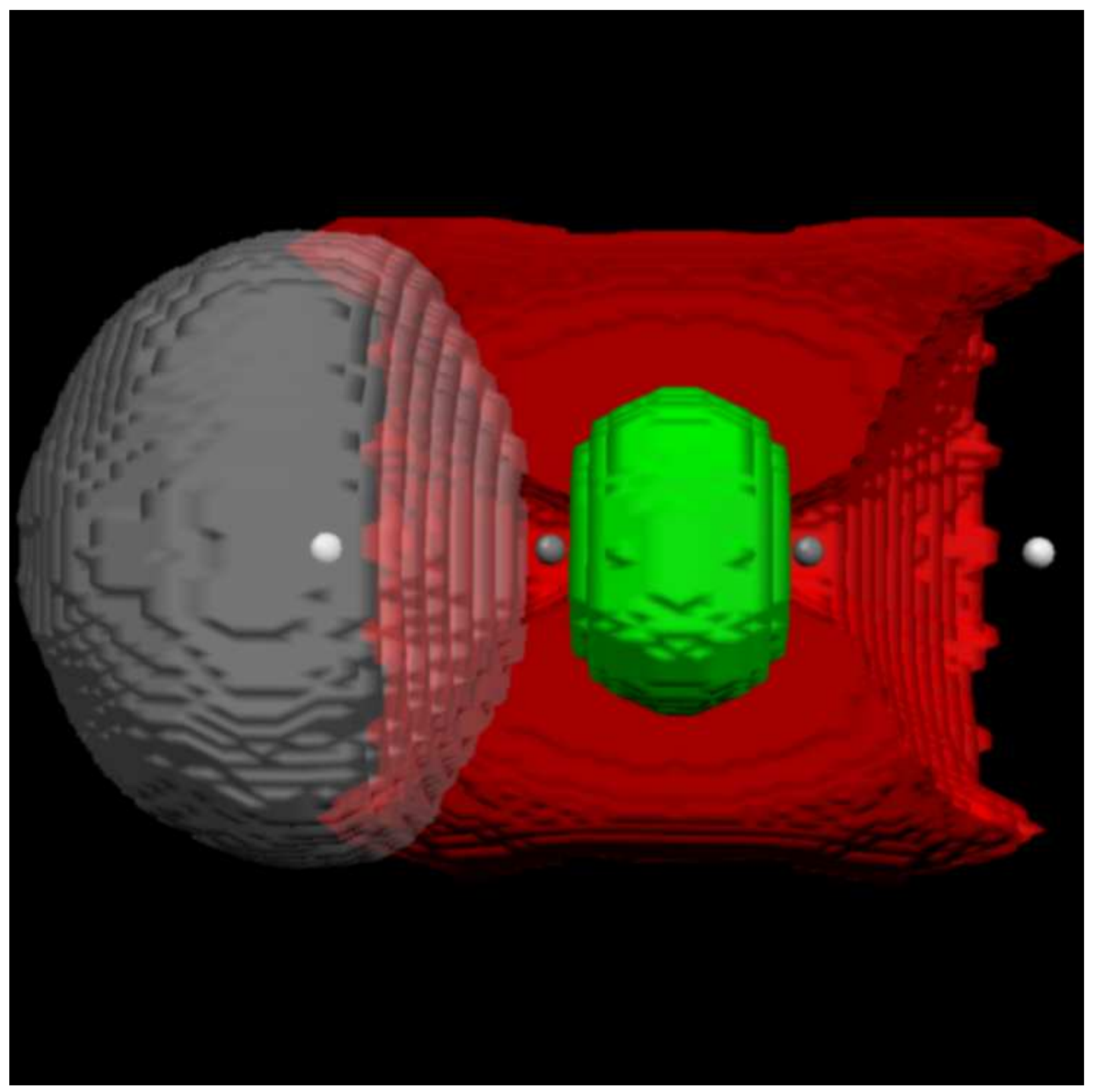

FIG. 11: 


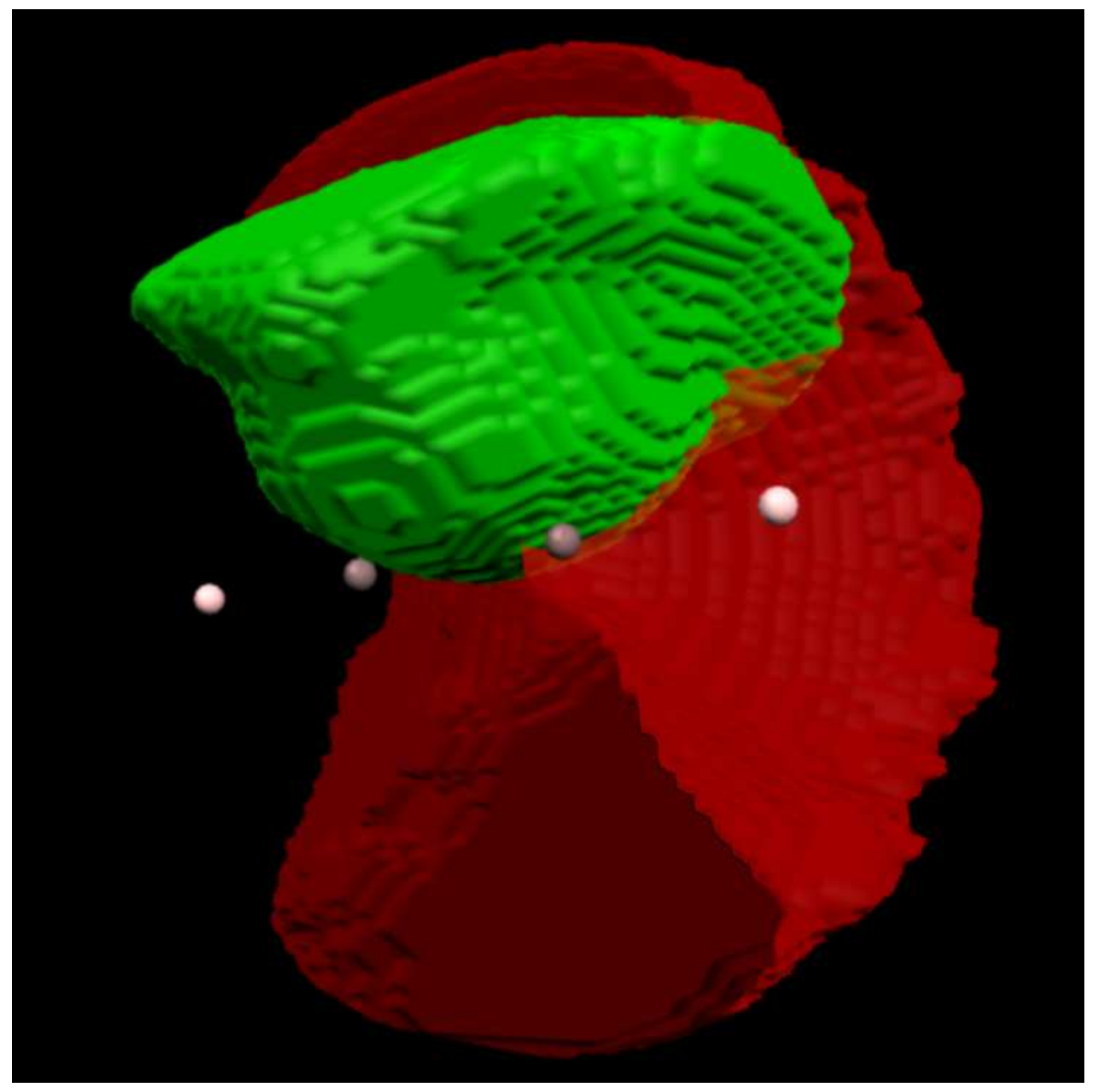

FIG. 12: 


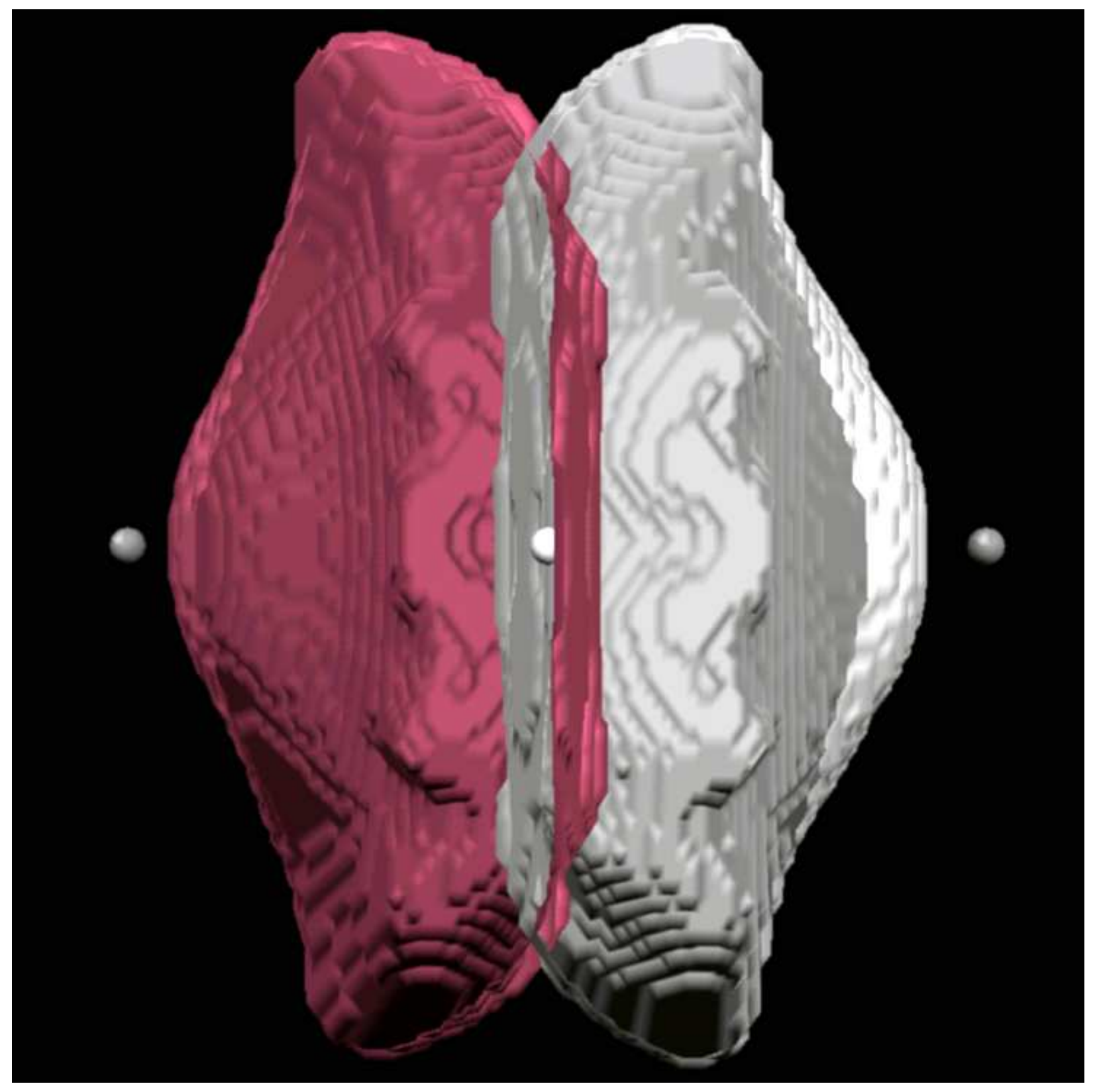

FIG. 13: 


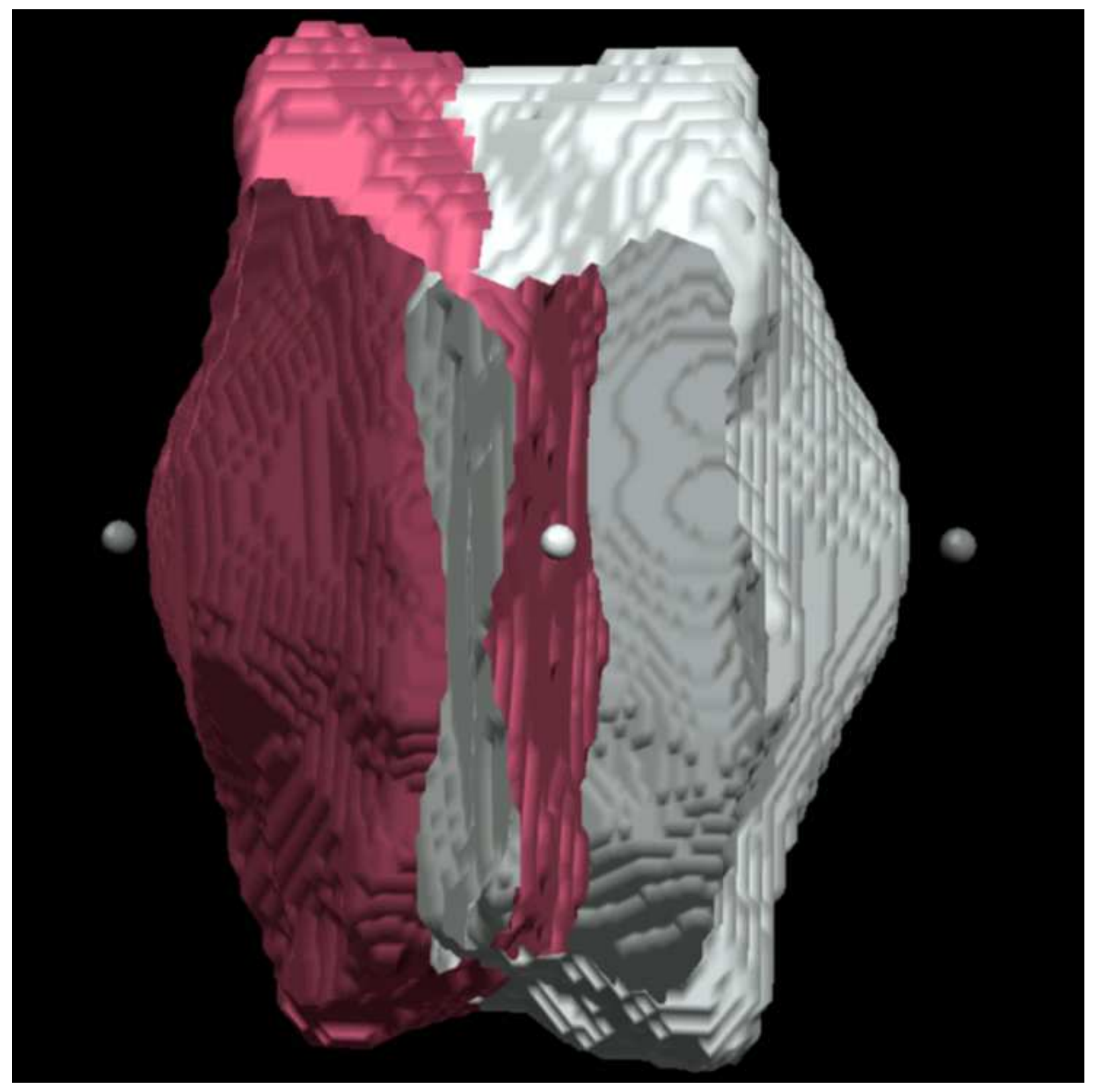

FIG. 14: 


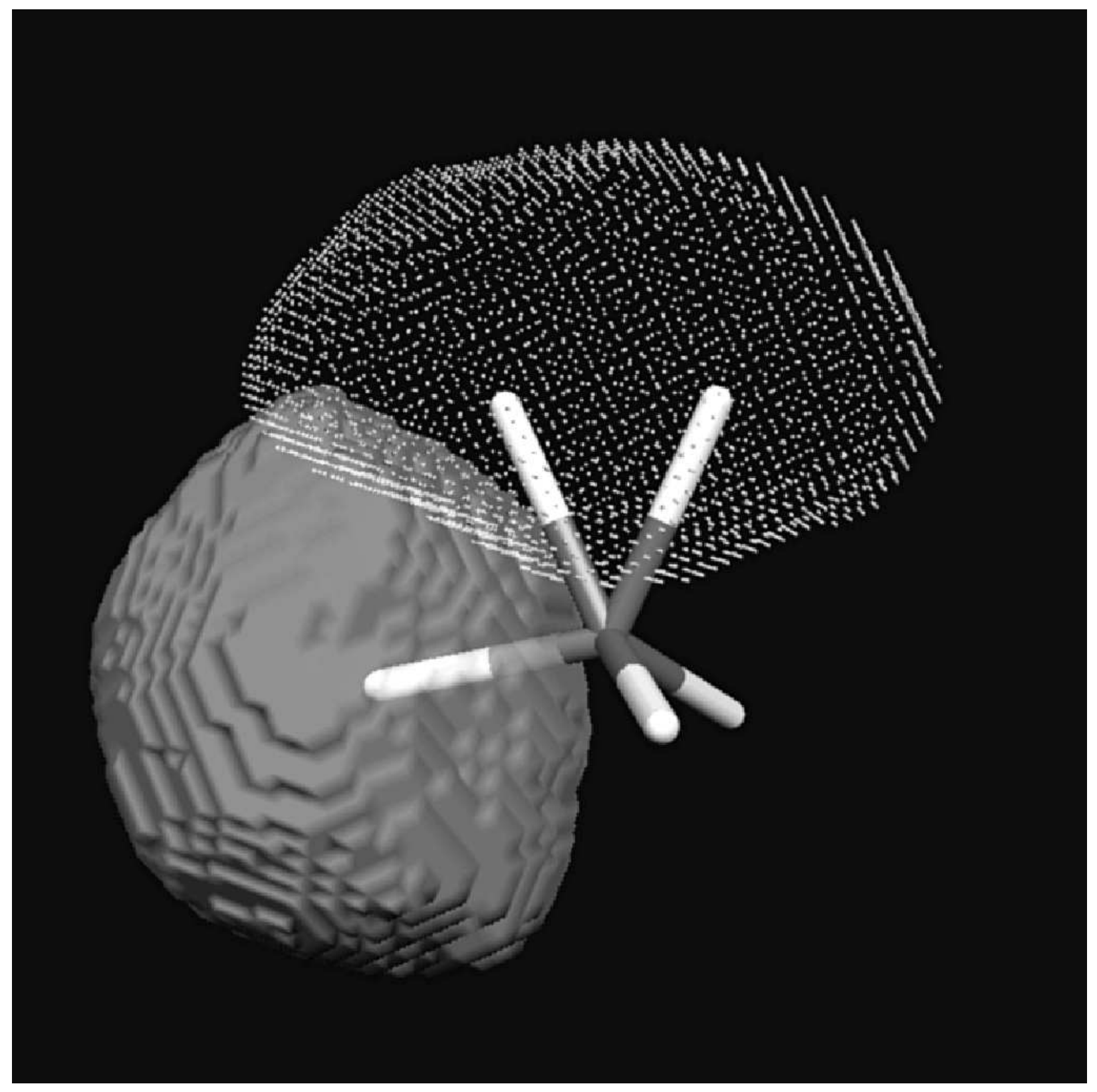

FIG. 15: 


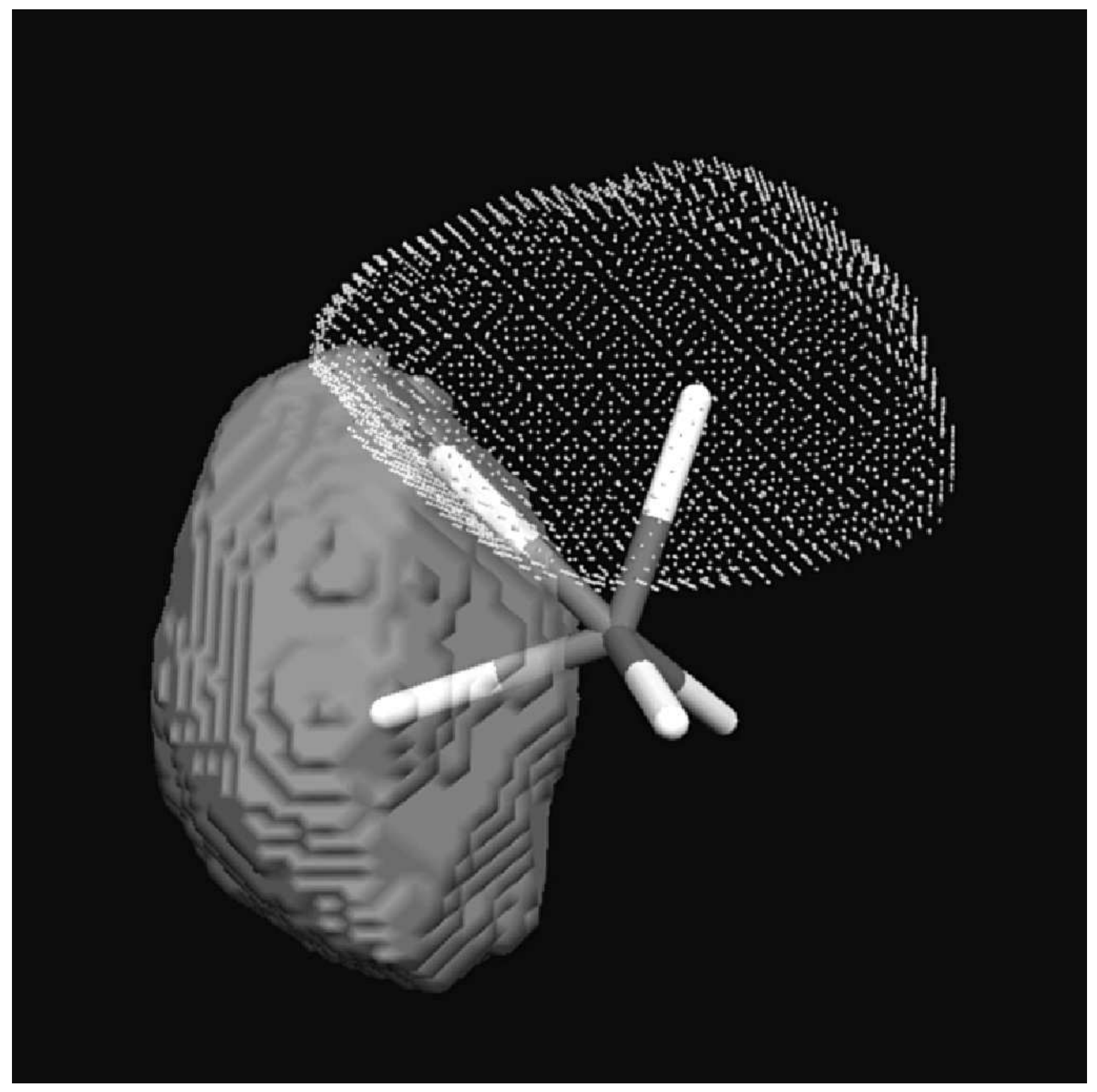

FIG. 16: 


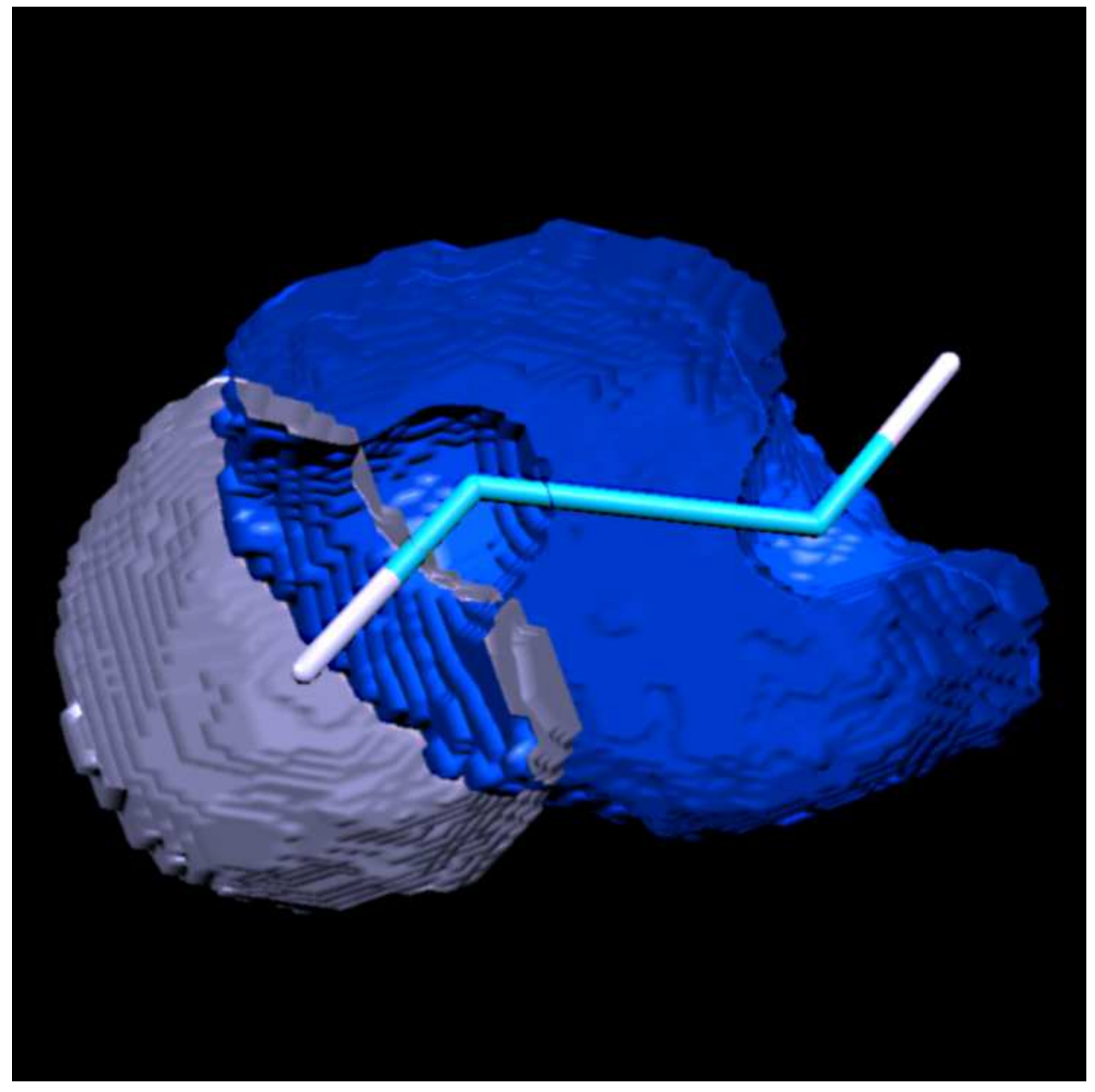

FIG. 17: 


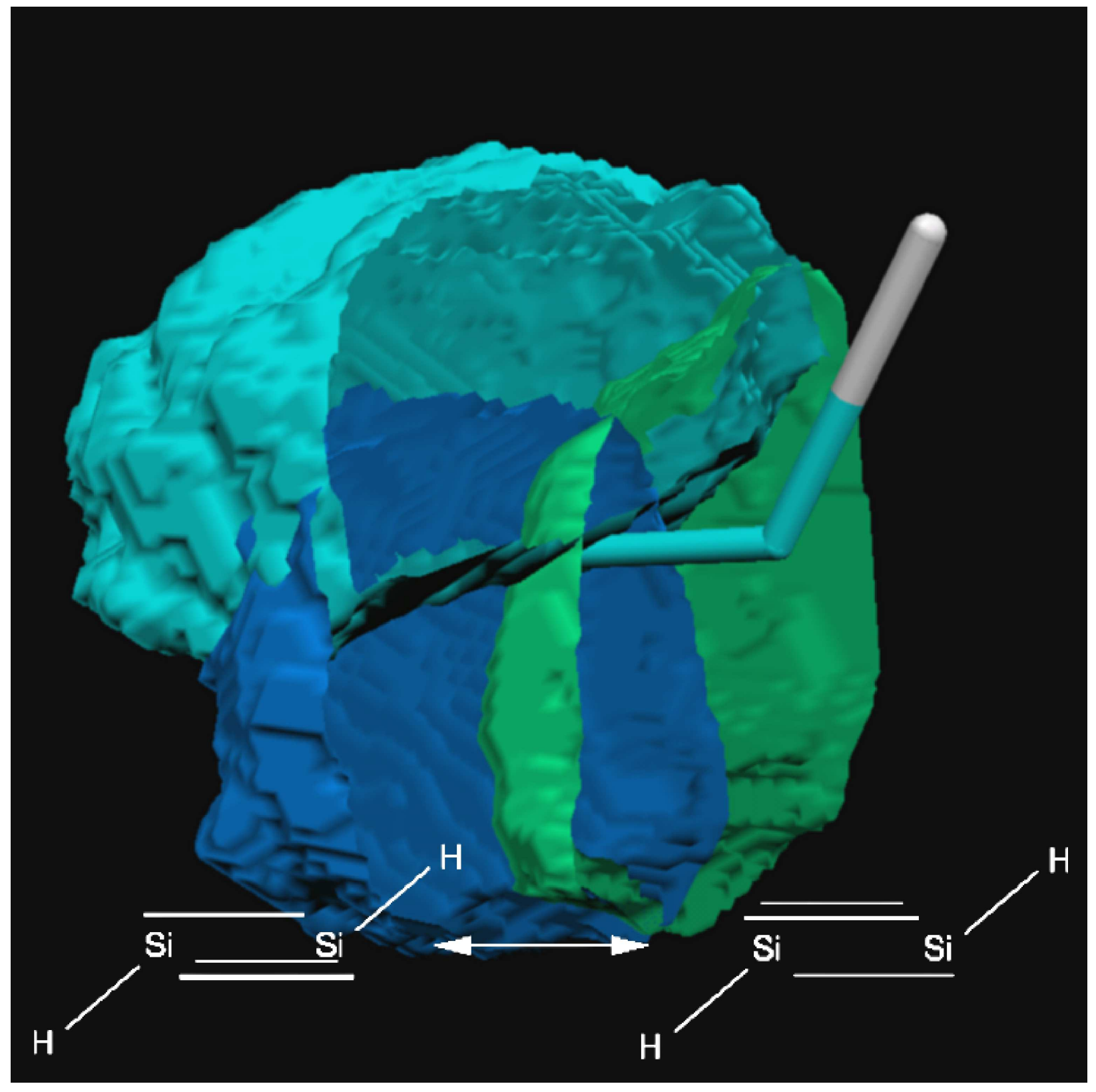

FIG. 18: 


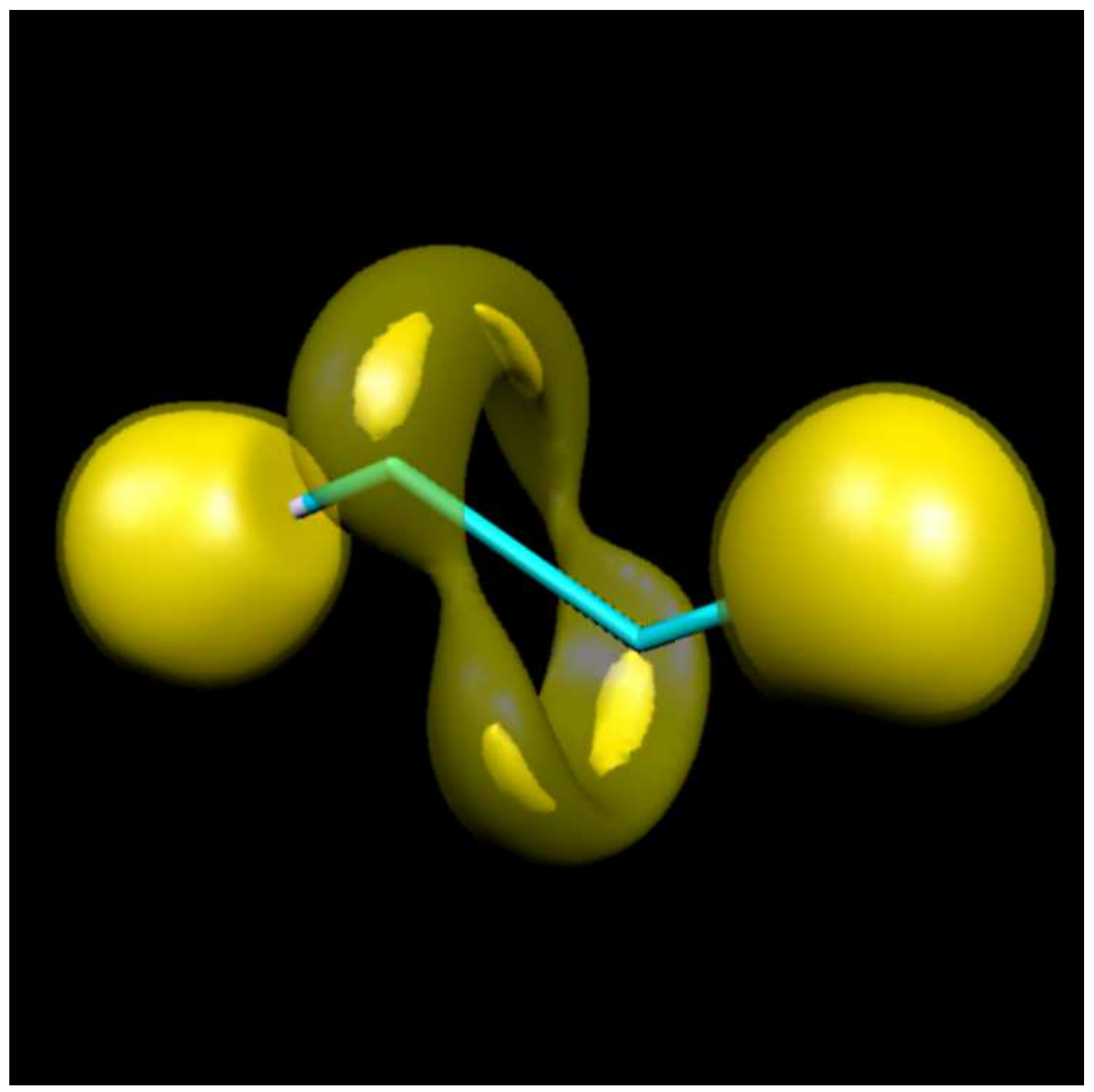

FIG. 19: 